This article was published as: Physical Review E 96 (6), 062604 (2017)

DOI: 10.1103/PhysRevE.96.062604 


\title{
Kinetics of doublet formation in bi-component magnetic suspensions: the role of the magnetic permeability anisotropy
}

\author{
M.T. Lopez-Lopez, ${ }^{1}$ F. Nogueras-Lara, ${ }^{1}$ L. Rodriguez-Arco ${ }^{1}$, N. Guigo ${ }^{2}$, \\ N. Sbirrazzuoli ${ }^{2}$, A.Yu. Zubarev ${ }^{3}$, S. Lacis ${ }^{4}$ and P. Kuzhir ${ }^{5 *}$ \\ ${ }^{1}$ Department of Applied Physics, University of Granada, Campus de Fuentenueva, 18071 Granada, Spain \\ ${ }^{2}$ University of Côte d'Azur, CNRS UMR 7272, Institute of Chemistry of Nice, Parc Valrose 06108 Nice, \\ France \\ ${ }^{3}$ Urals Federal University, Department of Mathematical Physics, Lenina Avenue 51, 620083 \\ Ekaterinburg, Russia \\ ${ }^{4}$ Faculty of Physics and Mathematics, University of Latvia, Zellu str. 23, RIGA, LV-1002, Latvia \\ ${ }^{5}$ University of Côte d'Azur, CNRS UMR 7010 Institute of Physics of Nice (INФNI), Parc Valrose, \\ 06108 Nice, France
}

\begin{abstract}
Micron-sized particles (microbeads) dispersed in a suspension of magnetic nanoparticles, i.e. ferrofluids, can be assembled into different type of structures upon application of an external magnetic field. This paper is devoted to theoretical modeling of a relative motion of a pair of microbeads (either soft ferromagnetic or diamagnetic) in the ferrofluid under the action of applied uniform magnetic field which induces magnetic moments in the microbeads making them attracting to each other. The model is based on a point dipole approximation for the magnetic interactions between microbeads mediated by the ferrofluid, however the ferrofluid is considered to possess an anisotropic magnetic permeability thanks to field-induced structuring of its
\end{abstract}

\footnotetext{
* Corresponding author e-mail: kuzhir@unice.fr
} 
nanoparticles. The model is tested against experimental results and shows generally better agreement with experiments than the model considering isotropic magnetic permeability of ferrofluids. The results could be useful for understanding kinetics of aggregation of microbeads suspended in a ferrofluid. From a broader perspective, the present study is believed to contribute to a general understanding of particle behaviors in anisotropic media.

\section{Introduction}

The design and control of the spatial organization of colloidal micron-sized particles (microbeads) with directional interactions appears as a powerful tool for the construction of novel structured materials which can be used in applications ranging from electronics to medical devices [1] In the particular case of magnetic particles, spatially addressable assemblies can be achieved by the application of non-contact magnetic fields, which induce a magnetic dipole within the particles, causing them to interact. However, this requires a positive, significant, response of the particles to the external field, such is the case of soft ferromagnetic particles or paramagnetic beads. This limitation can be overcome if the particles are dispersed in a carrier liquid which itself responds to the external field. In this way, diamagnetic particles, such as polymeric or silica beads as well as cells or living bacteria can be placed into programmed locations [2-3]. For example, assemblies of around a hundred diamagnetic and magnetic particles induced by microgradients of an external field in a paramagnetic liquid have been demonstrated [4]. Similar results can be obtained using a ferrofluid, i.e., a suspension of magnetic nanoparticles in a non-magnetic liquid, as the external medium. In practice, the ferrofluid behaves as a magnetic continuum in which the diamagnetic particles behave as magnetic holes and acquire an effective magnetic 
moment. Ferrofluid-mediated structures such as rotationally symmetric colloidal superstructures, flower and Saturn-ring arrangements, as well as crystalline phases and fractal aggregates have been reported [4-14].

Nevertheless, to design better controllable patterns of microbeads it is necessary to gain understanding of the physics of the interactions between particles which direct the assembly. In the case of ferrofluid-mediated assemblies, all the previous theoretical approaches are based on the assumption that the ferrofluid behaves as an isotropic magnetic continuum. This assumption is based on the fact that the ferrofluid particles are typically too small to overcome Brownian motion and create any anisotropic structure under an applied magnetic field, which would result in anisotropy of the ferrofluid magnetic permeability. Also, changes of the ferrofluid magnetic properties associated with variations of the ferrofluid concentration in the vicinity of the micronsized particles immersed into the ferrofluid are normally neglected. However, in previous works we have shown that the nanoparticles of the ferrofluid can undergo phase transitions such as condensation, especially around the dispersed soft ferromagnetic particles under the application of a magnetic field [15-17]. In the present paper, we demonstrate that the field-induced anisotropy of the magnetic permeability plays an important role in the computation of the magnetostatic force between the assembled microbeads. By doing so, the theoretical model is able to quantitatively reproduce the experimental trajectories and kinetics of doublet formation for pairs of either soft ferromagnetic (Ni) or diamagnetic (PMMA) microbeads in a ferrofluid when these form assemblies guided by the external field. The enhancement of the agreement between theory and experiments in comparison to a previous model [14] which 
considered an isotropic permeability constitutes a first step towards the development of more realistic models for the description of ferrofluid-directed assemblies.

From a general perspective, the studied system is a new example showing that the anisotropy of the suspending medium affects macroscopic behavior of suspended particles and (or) whole suspensions. A non-exhaustive list of such effects counts: (a) anisotropy of hydrodynamic drag of particles in a liquid crystal [18, 19]; (b) enhancement of the effective viscosity of a suspension of particles dispersed in a liquid crystalline polymer [20]; (c) anisotropy of optical trapping forces experienced by colloidal particles in anisotropic biological fluids [21]; anisotropic aggregation of colloidal particles in a nematic micellar solution [22]; rich phase behaviors in colloidal rod-sphere mixtures [23, 24]. From the macroscopic point of view, in most of the considered systems, the behavior of suspended spherical particles is governed by the anisotropy of suspending medium viscosity, while in the system considered in the present paper, the motion of microbeads is affected by the anisotropy of magnetic permeability. The results of the present paper are therefore believed to contribute to a general understanding of particle behaviors in anisotropic media.

\section{Materials and Methods}

As liquid carrier, we used a ferrofluid consisting of a suspension of magnetite nanoparticles dispersed in mineral oil of a dynamic viscosity $0.039 \mathrm{~Pa} \times \mathrm{s}$, prepared as described in [25]. The viscosity of the synthesized ferrofluid was $\eta=0.05$ Paxs as measured by a rotational rheometer Anton Paar Physica MCR 300. Stabilization against irreversible particle aggregation was achieved by steric repulsion via the adsorption of oleate ions on the particle surface. The particle size distribution was measured by 
dynamic light scattering with a Zetasizer instrument (Malvern Instruments, U.K.) and is shown in Fig. 1.

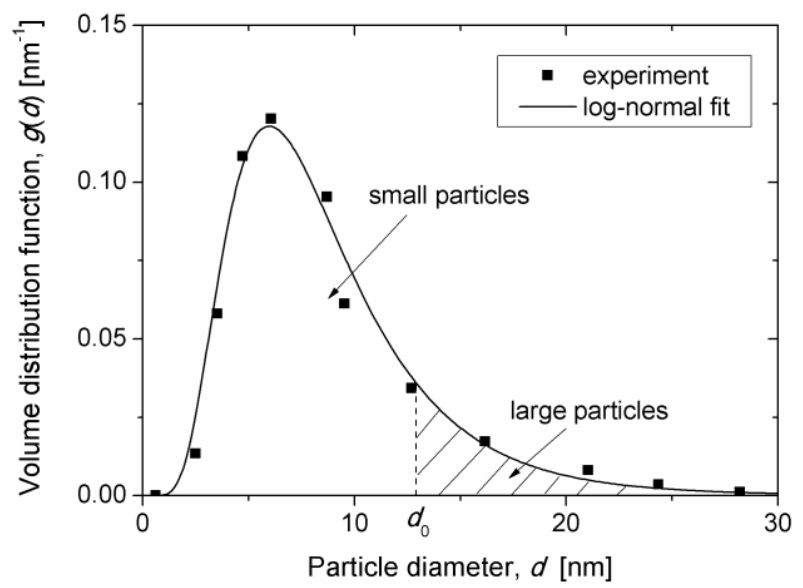

Fig. 1. Volume size distribution of ferrofluid nanoparticles. Squares correspond to the experimental curve and the solid line - to the log-normal fit. The relative volume occupied by the large nanoparticles, with diameter $d>d_{0}$ undergoing chain formation, with respect to the total volume of ferrofluid nanoparticles, corresponds to the hatched area below the curve. Numerical value of the threshold diameter $d_{0} \approx 13 \mathrm{~nm}$ is found in Appendix $\mathrm{C}$ by fitting the theoretical value of the ferrofluid initial magnetic permeability to the measured value $\mu=1.9$ [cf. Fig. 2].

The experimental curve [squares in Fig. 1] was fitted by the log-normal distribution [solid line in Fig. 1] with a mean value of the particle size $d_{m}=8.7 \mathrm{~nm}$ :

$$
g(d)=\frac{1}{\sqrt{2 \pi} \sigma d} \exp \left[-\frac{\ln ^{2}\left(d / d_{\mu}\right)}{2 \sigma^{2}}\right],
$$

where $\sigma=0.5$ and $d_{\mu}=7.67 \mathrm{~nm}$ are two adjustable parameters (standard deviation of the natural logarithm of the particle diameter and median diameter, respectively). The distribution function, as written in Eq. (1) is normalized to unity: $\int_{0}^{+\infty} g(x) d x=1$.

Magnetization curve of the ferrofluid was measured by a vibrating sample magnetometer (VSM 4500 EG\&G Princeton Applied Research, United States) and is shown in Fig. 2. The magnetization curve did not show any hysteresis confirming 
superparamagnetic behavior of ferrofluid nanoparticles. The fit of the magnetization curve by the Langevin law commonly admitted for ferrofluids [26] (even taking into account the particle size distribution [Eq. (1)]) gave a rather strong deviation between the fitted and actual initial slope of the magnetization curve, as can been seen in the inset of Fig. 2 [blue dashed curve for the Langevin fit]. Such discrepancy occurs likely because of chain-like structures formed under applied magnetic field, as will be discussed in Sec. IV-B and Appendix C. However, an empirical Fröhlich-Kennely relationship fitted much better to the experimental curve and reproduced relatively well the initial slope [solid red line in Fig. 2]. This relationship reads [27]:

$$
M(H)=\frac{\chi M_{S} H}{M_{S}+\chi|H|},
$$

where $H$ and $M$ are algebraic values of the magnetic field and magnetization inside the ferrofluid, respectively, $\chi=0.9$ and $M_{S}=26.9 \mathrm{kA} / \mathrm{m}$ are the two fitting parameters having the meaning of the initial magnetic susceptibility and saturation magnetization, respectively. The volume fraction, $\varphi$, of magnetic solids in the suspension was estimated by dividing the ferrofluid magnetization saturation $M_{S}$ by the magnetite particle magnetization saturation, $M_{S, p}=405 \mathrm{kA} / \mathrm{m}$ taken from the measurements in our previous work [25]. We obtained $\varphi \approx 0.066$ (6.6\% vol).

As assembling microbeads, we used soft ferromagnetic Ni powder (Merck KgaA) and diamagnetic poly(methyl methacrylate) (PMMA) powder (Spheromers CA10, Microbeads). The mean diameter and density of these particles were $5 \mu \mathrm{m}$ and $8.9 \mathrm{~g} \times \mathrm{cm}^{-3}$ for Ni powder, and $6 \mu \mathrm{m}$ and $1.2 \mathrm{~g} \times \mathrm{cm}^{-3}$ for PMMA powder. 


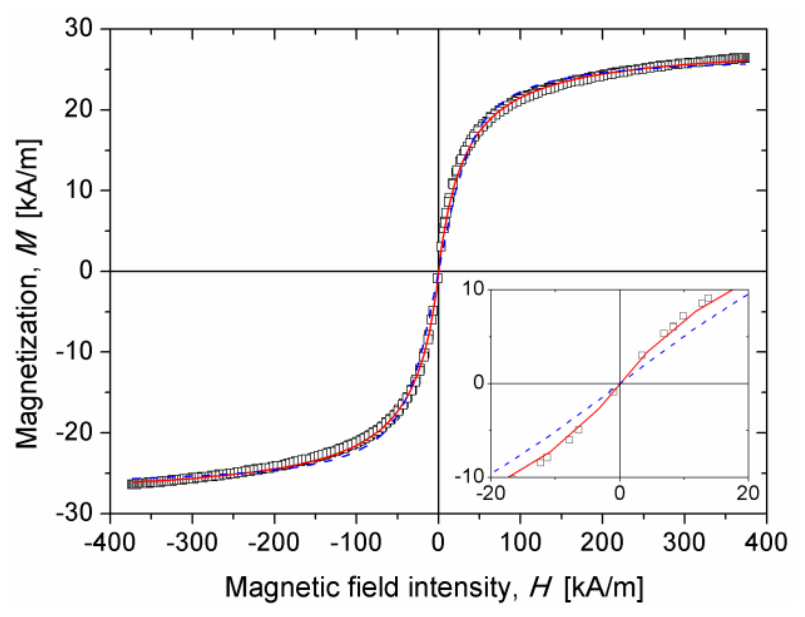

Fig. 2 (Color online). Magnetization curve of the ferrofluid. Points correspond to experimental data, red solid line - to the fit by Fröhlich-Kennely equation (2), blue dashed line - to the fit by the Langevin law [26]. The inset shows a zoomed view of the magnetization curve at weak magnetic fields.

We prepared three different kinds of suspensions of microbeads in the ferrofluid carrier: (i) suspensions of soft ferromagnetic (Ni) microbeads in the ferrofluid known as bi-disperse magnetorheological fluids [28]; (ii) suspensions of diamagnetic (PMMA) microbeads in the ferrofluid, known as inverse ferrofluids [29]; (iii) suspensions of Ni and PMMA microbeads in the ferrofluids, known as inverse magnetorheological fluids [30]. Note that soft ferromagnetic microbeads typically have a negligibly narrow magnetization hysteresis and their macroscopic magnetic behavior is described in the same way (i.e. by magnetic permeability and magnetization saturation) as that of paramagnetic microbeads, such as polystyrene beads with embedded superparamagnetic iron oxide nanoparticles, frequently used in biological analyses. This means that all results of this work will be identical for soft ferromagnetic and paramagnetic beads having the same magnetic permeability. The solid concentration of microbeads in the final suspensions was very low (of the order of 0.01 vol.\%), so that it was possible to find isolated pairs of microbeads to monitor their dynamic under magnetic field application. 
We placed a drop of the suspensions between two microscope glass slides, separated by glass spacers with an approximate thickness of $0.13 \mathrm{~mm}$. We used an optical microscope (Thermo Fisher Scientific, USA) connected to a CCD camera to investigate the kinetics of doublet formation for a chosen pair of particles. For this aim, we located a pair of stationary particles. Afterwards, we applied a magnetic field in the perpendicular direction to the axis of the microscope with the help of a pair of Helmholtz coils, and monitored the particle trajectories as a function of time. The intensity of the applied magnetic field at the focal point of the microscope was approximately homogeneous with a value of $H_{0}=10 \mathrm{kA} / \mathrm{m}$, as measured with a teslameter.

\section{Experimental results}

For pairs of microbeads close enough (at distances less than about ten microbead diameters) so that the magnetostatic interaction between them was not negligible, the equilibrium state was reached with the microbeads in contact (Fig. 3). Theoretically, there is an exception to this when the initial center-to-center line forms an angle of 90 degrees with respect to the direction of the applied magnetic field for similar microbeads (Ni-Ni or PMMA-PMMA), or 0 degrees for dissimilar microbeads (NiPMMA). In this case it is expected that microbeads are continuously repelled following a straight line. However, this situation is very difficult to be found from the experimental point of view, since it is an unstable equilibrium that is altered by any minimal perturbation giving rise to attraction. Therefore, in our experiments we always obtained equilibrium states with the particles in close contact (Fig. 3). For pairs of similar particles, equilibrium was reached with the particle center-to-center line parallel to the direction of the applied magnetic field (Figs. 3b and 3d). On the other hand, for 
pairs of dissimilar particles, the particle center-to-center line was perpendicular to the applied field direction in the equilibrium configuration (Fig. 3f). This is explained by opposite mutual orientation of the apparent magnetic moments of dissimilar microbeads (collinear with applied field for soft ferromagnetic nickel particle and anti-collinear for diamagnetic PMMA particle placed into the ferrofluid), in which case the ground state is achieved when the center-to-center line of the pair lies perpendicularly to the applied field [14].

To monitor the particle position as a function of time after application of the magnetic field we tracked the particle trajectories using the center of the particles as reference points.

(a)

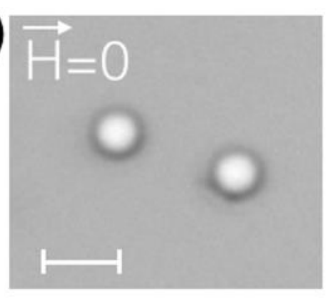

(b)



(c)

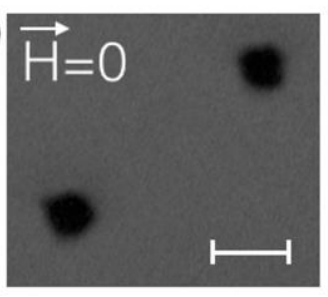

(d)

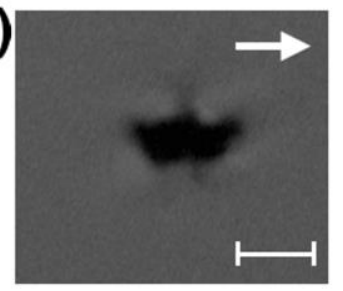

(e)

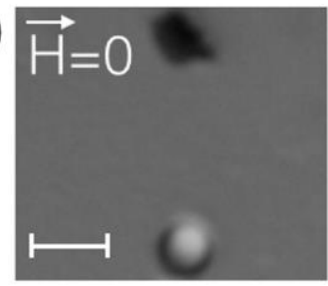

(f)

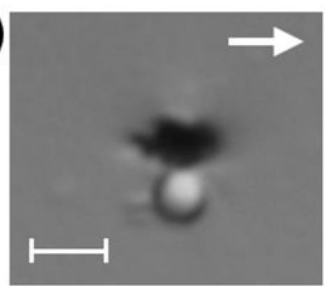

Fig. 3. Some snapshots illustrating the dynamics upon magnetic field application of pairs of microbeads dispersed in a ferrofluid: (a-b) two diamagnetic particles (white spheres), (c-d) two soft ferromagnetic particles (black spheres), (e-f) a soft ferromagnetic particle (black sphere) and a diamagnetic particle (white sphere). Snapshots shown in (a,c,e) were taken before magnetic field application (zero time). Snapshots shown in $(b, d, f)$ represent the equilibrium state. The white arrows indicate the magnetic field direction. Bar length: 5.5 microns. 


\section{Theory and discussion}

\section{A. General framework}

In our previous work we developed a theoretical model that computed the magnetostatic force between microbeads by means of the dipole-dipole approximation, as well as the friction force via a Rayleigh dissipation function [14]. This simple model described reasonably well the experimental trajectories from the qualitative point of view. However, the discrepancy from the quantitative point of view between the experimental and theoretical trajectories was significant, as evidenced by Figure 5 of ref. 14. The objective of the present work was to find the physical origin of this discrepancy. First of all, we considered the multipolar theory in contrast to the dipolar approximation. As discussed in Appendix A we did not find any significant improvement by using the multipolar theory because multipolar magnetic interactions become significant only at close distance between neighboring microbeads (the gap between microbead surfaces typically lower than microbead radius). On the contrary, we found that the anisotropy of the magnetic permeability may be the cause for the discrepancy between predictions of our simple dipole-dipole model and the experimental findings.

The basic idea consists in supposing that the ferrofluid in which the microbeads are dispersed may undergo a field induced structuring manifested by the appearance of nanoparticle chains or drop-like elongated aggregates extended along the direction of the applied magnetic field $[15,31,32]$. Such a structuring is expected to result in anisotropy of the magnetic properties of the ferrofluid, as well of the hydrodynamic mobility of the microbeads immersed in an anisotropic ferrofluid. The magnetic 
permeability $\mu_{z}$ and the microbead mobility $b_{z}$ along the structures are expected to be higher than the magnetic permeability $\mu_{x}$ and mobility $b_{x}$ in transverse direction, as depicted schematically in Fig.4a. Such anisotropy is expected to change magnetic and hydrodynamic drag forces acting on microbeads and consequently the trajectories of their mutual approach, as compared to those in an isotropic ferrofluid [Fig. 4b]. In experiments, we do not clearly see appearance of bulk micron-sized aggregates in the ferrofluid, but this does not exclude appearance of chain-like aggregates, predicted for relatively low nanoparticle concentrations and magnetic fields [33, 34].

(a)

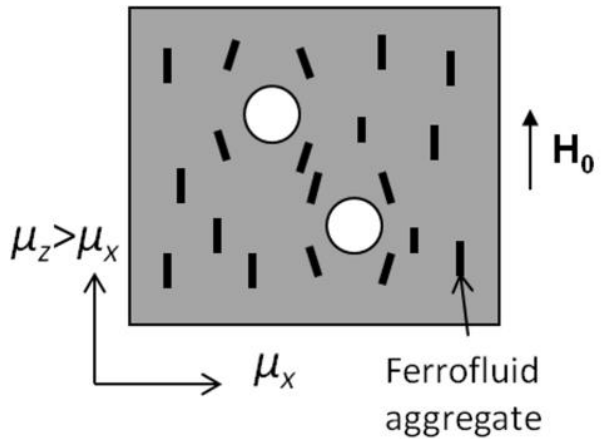

(b)

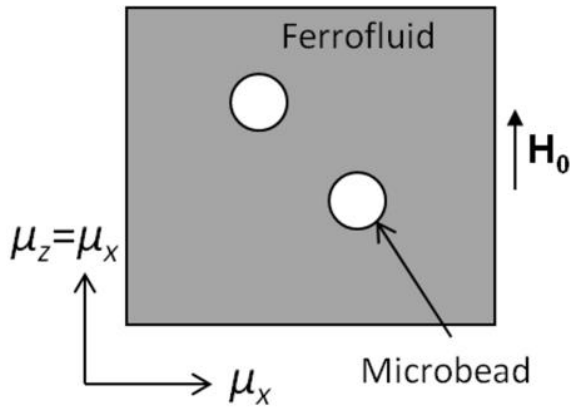

Fig. 4. A sketch of the ferrofluid with two immersed microbeads. In the case (a) the applied external magnetic field (of intensity $H_{0}$ ) induces field aligned aggregates of ferroluid nanoparticles, whose size is exaggerated. These aggregates result in anisotropic magnetic permeability with the largest value $\mu_{z}-$ along the aggregates, thus along the applied field and the minor value $\mu_{x}$-across the aggregates, thus perpendicularly to the applied field. In the case (b) there is no structuring in the ferrofluid and the magnetic permeability is isotropic.

These single chains are typically composed of a dozen of nanoparticles and therefore invisible in optical microscope. In what follows we will check how the anisotropy of mobility and of magnetic permeability affects the kinetics of the formation of doublets of microbeads immersed in the ferrofluid. 
At the first approximation, the field-induced anisotropy could be taken into account assuming that all nanoparticle chains have the same length and are oriented along the lines of the external uniform magnetic field $\mathbf{H}_{0}$. This implies that the magnitudes $\mu_{x}, \mu_{z}$ and $b_{x}, b_{z}$ are constant over the ferrofluid volume. Such homogeneous distribution takes place far enough from both microbeads where the magnetic field is homogeneous. However, neglect of possible deviation of the chains from the direction of $\mathbf{H}_{0}$ could generate unphysical results. The aforementioned approximation can only be used, after appropriate modifications, in some limiting cases considered below:

(a) Magnetic torque acting on nanoparticle chains is much larger than the hydrodynamic torque induced by local velocity fields around the microbeads. In this case, the nanoparticle chains are everywhere directed along the magnetic field lines. The average chain length is an increasing function of the local magnetic field, thus it changes from point to point in the vicinity of the microbeads following the magnetic field map. Whatever the direction of the external magnetic field $\mathbf{H}_{0}$, the chains will adopt the direction of the local magnetic field $\mathbf{H}$ around the microbeads and the local ferrofluid magnetization $\mathbf{M}$ will be collinear with the local magnetic field in each ferrofluid point. This collinearity implies local isotropy of the ferrofluid magnetic permeability, which will be independent of the orientation of the external magnetic field $\mathbf{H}_{0}$ with respect to the microbead. Analysis of the magnetic field distribution around microbeads and of the ferrofluid magnetization shows that the magnetic field variation around microbeads produces only a few percent spatial variation in the ferrofluid magnetic permeability at the distance larger than one microbead radius from its surface. At this condition, the magnetostatic potential $\Psi$ of the local magnetic field is defined by the Laplace equation, $\nabla^{2} \Psi=0$ and differs from the potential in isotropic ferrofluid by a 
constant numerical factor. Using point dipole approximation, it is easily shown that the magnetic force between microbeads will have the same orientation in the structured and unstructured ferrofluids and the field-induced change of the magnetic permeability will not influence the particle trajectories. The field-induced structuring will however induce anisotropy of the microbead mobility because, at fixed orientation of the external field, $\mathbf{H}_{0}$, the mutual orientation between chains and the bead velocity $\mathbf{v}$ changes when the orientation of $\mathbf{v}$ changes. The chains are in average oriented along $\mathrm{H}_{0}$ direction (along $z-$ axis), so that $b_{z}>b_{x}$. It can be easily shown, that higher mobility $b_{z}$ along $z$ axis shifts the bead trajectory apart from the $z$-axis with respect to the case of isotropic ferrofluid, in contrast to experimental findings.

(b) Magnetic torque acting on nanoparticle chains is much smaller than the hydrodynamic torque. In this case, the nanoparticle chains are everywhere oriented along the fluid streamlines around moving microbeads. The orientation distribution will be invariant with respect to direction of the microbead motion, so that the bead mobility will be isotropic in this case. However, the chains make now some angle with the local magnetic field vector $\mathbf{H}$ resulting in flow-induced local anisotropy of the ferrofluid magnetic permeability. The average ferrofluid magnetization near the moving microbead will change with orientation of the external field $\mathbf{H}_{0}$, thus inducing anisotropy of the mean magnetic permeability averaged over the ferrofluid volume, at least at the length scale of hydrodynamic perturbation around moving microbeads.

As, we will see later in Sec. IV-C, average anisotropic magnetic permeability allows correct predictions of the bead trajectories. However, we have to prove that our experimental conditions fit to the limiting case (b) and, to this purpose, in the next 
section IV-B, we will estimate hydrodynamic and magnetic torques exerted to nanoparticle chains, as well as the chain orientation and length.

\section{B. Estimation of the chain orientation and length}

The magnetic and hydrodynamic torques strongly depend on the chain length. First, we have to estimate the mean number of ferrofluid nanoparticles $\langle N\rangle$ per chain, called hereinafter the chain length, and then apply the torque balance to estimate the chain orientation.

Short chains containing several nanoparticles are subject to strong Brownian motion; their translational Péclet number is estimated to be of the order of $P e \sim 10^{-2}$. At the first glance, their length is expected to be unaffected by fluid motion but governed by local thermodynamic equilibrium. Analysis of the average equilibrium chain length (detailed in Appendix B), shows that

(a) At the given magnetic field, $H_{0}=10 \mathrm{kA} / \mathrm{m}$ and nanoparticle volume fraction, $\varphi=0.066$ (6.6\%vol.), the average number $\langle N\rangle$ of nanoparticles per chain is only about 1.05 if the average nanoparticle diameter $d_{m}=8.7 \mathrm{~nm}$ is taken for calculations;

(b) Since the ferrofluid nanoparticles are polydisperse [Fig. 1], only the largest particles with a diameter larger than some threshold value $d_{0}$ are expected to form chains. The value $d_{0}$ divides the particle size distribution onto the right region of "large nanoparticles" [hatched area in Fig. 1] and the left region of "small nanoparticles" having a mean diameter and a volume fraction equal to $d_{L}, \varphi_{L}$ and $d_{S}, \varphi_{S}$, correspondingly, with $\varphi_{L}+\varphi_{S}=\varphi$. Since theoretical evaluation of the values $d_{0}$ or $d_{L}$ are related to some difficulties, we obtain them by fitting the theoretical value of the 
ferrofluid initial magnetic permeability to the measured value $\mu=1+\chi=1.9$. To this purpose we develop a bi-disperse model of the ferrofluid considering the fraction of small particles, all having the same diameter $d_{S}$ and the fraction of large particles with the diameter $d_{L}$ [Appendix B]. Then, this model is applied for magnetic permeability calculations [Appendix C] and the single adjustable parameter, the average diameter of large nanoparticles is found to be equal to $d_{L} \approx 17 \mathrm{~nm}$ corresponding to the fraction of large particles of $\varphi_{L} / \varphi \approx 0.15$. These values of the size and volume fraction of the large particles give the average chain length $\langle N\rangle=5.4$ [Appendix B], which will be kept for subsequent calculations.

Before estimation of the chain orientation, we have to assess the importance of Brownian motion leading to orientational fluctuations. To this purpose, we evaluate the rotational Péclet number in the limit of large aspect ratio chains $(\langle N\rangle>>1)$ [35]: $P e_{r} \sim$ $\eta \dot{\gamma} d_{L}^{3}\langle N\rangle^{3} /\left(k_{B} T\right)$, where $k_{B} T \approx 4 \times 10^{-21} \mathrm{~J}$ is the thermal agitation energy, $\eta$ is the viscosity of the liquid carrier of the ferrofluid and $\dot{\gamma} \sim v / a$ is a characteristic value of the rate-of-strain tensor corresponding to the flow around the microbeads of a radius $a$ moving at a velocity $v$. This velocity scales as $v \sim F_{m} /(\eta a)$, as estimated from the Stokes drag, where the magnetic force between microbeads scales as $F_{m} \sim \mu_{0} H_{0}{ }^{2} a^{2}$, where $\mu_{0}=4 \pi \times 10^{-7} \mathrm{H} / \mathrm{m}$ is the magnetic permeability of vacuum. This gives $\eta \dot{\gamma} \sim \mu_{0} H_{0}{ }^{2}$ and the rotational Péclet number is of the order of $P e_{r} \sim \mu_{0} H_{0}{ }^{2} d_{L}{ }^{3}\langle N\rangle^{3} /\left(k_{B} T\right) \approx 20$. This 
allows us to neglect angular fluctuations ${ }^{\dagger}$ of chains and estimate the chain orientation under flow and magnetic field from equilibrium of magnetic and hydrodynamic torques acting on nanoparticle chains.

These torques, as well as their ratio can be estimated as follows [36]: $T_{m} \sim$ $\mu_{0} H_{0}{ }^{2}\langle N\rangle\langle d\rangle^{3} ; T_{h} \sim \eta \dot{\gamma}\langle N\rangle^{3}\langle d\rangle^{3}$ and $T_{h} / T_{m} \sim\langle N\rangle^{2} M a$, where $M a=\eta \dot{\gamma} /\left(\mu_{0} H_{0}{ }^{2}\right)$ is the Mason number, which is of the order of unity, $M a \sim 1$, that is simply explained by the fact that the microbead motion with an inherent drag force is induced by the magnetic attraction between microbeads, so that the drag force is of the order of magnitude of the magnetic force. This gives the following estimation for the torque ratio: $T_{h} / T_{m} \sim$ $\langle N\rangle^{2} M a \approx 30$. This shows that the hydrodynamic torque is much larger than the magnetic one allowing us to confirm that the chains are expected to be mostly aligned along the streamlines around the moving microbeads.

Even though the translational Péclet number of individual nanoparticles is much less then unity, $P e<<1$, the hydrodynamic forces acting on chains may appear to be of the same order of magnitude that the magnetic forces in the same way as hydrodynamic and magnetic torques. We have therefore to check whether the chains are destroyed by the flow. We can check it for the most severe shear flow occurring in the vicinity of the moving microbead surface. Since the flow at the bead surface is a simple shear and the magnetic field is almost normal to the bead surface (in the case of highly magnetic microbeads), the problem reduces to the well-known case of dilute electro- or

\footnotetext{
$\dagger$ Neglecting rotational Brownian motion of chains does not contradict to important translational Brownian motion of single nanoparticles. The first one is described by rotational Péclet number $P e_{r}$ and the second one - by translational Péclet number $P e$, with $\left.P e_{r} \sim P e<N\right\rangle^{3}$, such that we have $P e_{r}>1$ even for $P e<<1$ providing that $\left\langle N>^{3} \approx 160\right.$.
} 
magnetorheological suspensions with the chain length calculated from the coupled equations of the torque and force balance [36]. Adapting this calculation to the case of the chains of monodomain nanoparticles considered as rigid dipoles (the magnetic anisotropy energy is much higher than the thermal agitation energy $k_{B} T$ at particle size $d_{L} \approx 17 \mathrm{~nm}$ ), we get the angle between the flow and the chain $\Theta \sim \chi_{L}^{-1 / 2}$ and the chain length, at which the hydrodynamic force break it, is of the order of $\langle N\rangle \sim \chi_{L}^{3 / 4} M a^{-1 / 2} \sim$ $\chi_{L}^{3 / 4} \approx 17$, where $\chi_{L} \approx 8 \lambda \approx 43$ is the initial magnetic susceptibility of large nanoparticles and the dipolar coupling parameter $\lambda$ given by Eq. (B-1d) in Appendix B is calculated for the average size $d_{L} \approx 17 \mathrm{~nm}$ of the nanoparticles constituting the chains. This calculation confirms that the chains make a small angle $\Theta<<1$ with the flow and shows that that the chains are not ruptured by the flow because their average equilibrium length $\langle N\rangle \approx 5.4$ is lower than the value $\langle N\rangle \approx 17$ obtained from the force balance.

\section{Model of homogeneous anisotropic magnetic permeability}

Having proved that the flow around the approaching microbeads orients the nanoparticle chains suspended in the ferrofluid along the streamlines and that this causes anisotropy of the ferrofluid magnetic permeability but not of the particle mobility, we can proceed now to calculations of the microbead trajectories. Local values of the components $\mu_{x}$ and $\mu_{z}$ of the magnetic permeability tensor should depend on the mutual orientation between the local field $\mathbf{H}$ and streamlines and therefore should vary from point to point in the ferrofluid volume. Moreover, since different trajectories make different angles with the external field $\mathbf{H}_{0}$, volume average values of $\mu_{x}$ and $\mu_{z}$ will 
depend on the trajectory shape. Analytical solution of this problem is impossible. However, to capture the physical picture of the anisotropy effect, we can consider two extreme cases when the microbead motion is slightly deviated from the magnetic field direction ( $z$ axis in Fig. 4a) or slightly deviated from the direction of $x$ axis. In the first case, the microbead motion will in average orient the chains along the applied field and we will have $\mu_{z}>\mu_{x}$. In the second case, the beads will orient the chains transversely to the applied field and we will have $\mu_{z}<\mu_{x}$. For these two cases, we will neglect spatial variations of both components $\mu_{z}$ and $\mu_{x}$, thus consider them constant in all points of the ferrofluid. The values $\mu_{z}$ and $\mu_{x}$ are estimated assuming a perfect alignment of the chains along either $z$ or $x$ axes. We obtain the value 1.90 for the permeability in the direction of chains from direct magnetization measurements [Sec. II]. The permeability value in the direction transverse to the chains is estimated in Appendix $\mathrm{C}$ and is equal to 1.38 such that the ratio $\hat{\mu}=\mu_{z} / \mu_{x}$ is approximately equal to either 1.4 or 0.7 depending on the chain orientation. As we shall see at the end of this Section, the magnitude $\hat{\mu}$ is the main parameter describing the ferrofluid anisotropy. In what follows, we describe in detail the calculations of the bead trajectories under above considered assumptions.

Let us consider two magnetizable spherical particles; let $\mathbf{r}_{1}$ and $\mathbf{r}_{2}$ be the radiusvectors of their centers. In the inertialess approximation, which, as a rule, is fulfilled for micron-sized particles moving in a viscous liquid, we get:

$$
\frac{d \mathbf{r}_{1}}{d t}=-\mathbf{b}_{1} \cdot \mathbf{F}(\mathbf{r}), \quad \frac{d \mathbf{r}_{2}}{d t}=\mathbf{b}_{2} \cdot \mathbf{F}(\mathbf{r}), \quad \mathbf{r}=\mathbf{r}_{2}-\mathbf{r}_{1}
$$

Here $\mathbf{F}$ is the magnetic force that the first particle exerts on the second one, $\mathbf{b}_{1}$ and $\mathbf{b}_{2}$ are the tensors of the particle hydrodynamic mutual mobility. In general terms, their 
components depend on the distance $r$ between the particles [37]. When the distance increases, the tensors $\mathbf{b}_{1,2}$ tend to the Stokes scalar mobilities:

$$
b_{1,2}=\frac{1}{6 \pi \eta a_{1,2}}
$$

where $a_{1}$ and $a_{2}$ are the particles radii. To the best of our knowledge, the explicit analytical forms of the tensors $\mathbf{b}_{\mathrm{i}}$, valid for the arbitrary magnitudes of $r$, are unknown. Some useful numerical data can be found in [38]. These results show that the dependence of the components of $\mathbf{b}_{\mathrm{i}}$ on $r$ is significant when the gap between the particles is much smaller than their diameters. The limiting formula given by Eq. (4) leads to a good approximation when the distance $r$ is as large as $2.5 a$ or longer. Combining the terms of Eq. (3), we get the equation for the radius-vector $\mathbf{r}$ :

$$
\frac{d \mathbf{r}}{d t}=\left(b_{1}+b_{2}\right) \mathbf{F}(\mathbf{r})
$$

In order to calculate the trajectories (i.e., solving Eq. (5)) of the microbeads, we have to find the magnetic force between them. We calculate the force using a pointdipole limit, taking into account the anisotropy of the suspending liquid (ferrofluid). The magnetic force derives from the potential energy of the interaction between the microbeads, and, in the point-dipole limit, this energy can be calculated as follows:

$$
U=-\mathbf{m}_{2} \cdot \mathbf{H}_{1}
$$

where $\mathbf{m}_{2}=m_{2} \mathbf{e}_{z}$ is the dipole moment of particle 2 and $\mathbf{H}_{1}$ is the magnetic field induced by the dipole moment $\mathbf{m}_{1}$ of the particle " 1 " at the location of the particle " 2 ". The force $\mathbf{F}$ can be calculated from Eq. (6) using the general relationship: 


$$
\mathbf{F}=-\nabla U
$$

In the point-dipole limit the magnetic moments $\mathbf{m}_{1}$ and $\mathbf{m}_{2}$, as well as the induced magnetic fields $\mathbf{H}_{1}$ and $\mathbf{H}_{2}$ are considered to be unaffected by the presence of the neighboring particle. Thus the induced field $\mathbf{H}_{1}$ can be simply calculated as the field of a point dipole m1 placed into an unbounded anisotropic magnetic medium.

Using an electrostatic analogy, the magnetostatic potential of a dipole $\mathbf{m}_{\mathbf{1}}$ can be calculated as the sum of the magnetostatic potentials $\varphi^{+}$and $\varphi^{-}$of two equivalent charges " $+q$ " and " $-q$ " situated at an infinitely small distance $2 d$ from each other (Fig.5). Following the reasoning of Landau and Lifshitz [39], we introduce the Cartesian reference frame $\left(x^{\prime}, y^{\prime}, z^{\prime}\right)$, where $x^{\prime}=x / \sqrt{\mu_{x}}, \quad y^{\prime}=y / \sqrt{\mu_{y}}, \quad z^{\prime}=z / \sqrt{\mu_{z}}$. The magnetostatic potential of the point charge $q$ located at the origin reads:

$$
\phi=\frac{q}{4 \pi \mu_{0} \sqrt{\mu_{x} \mu_{y} \mu_{z}} r^{\prime}}
$$

where $r^{\prime 2}=x^{\prime 2}+y^{\prime 2}+z^{\prime 2}$. Thus, the magnetostatic potential of the point dipole [Fig. 5] is:

$$
\begin{aligned}
& \phi_{d}=\phi^{+}+\phi^{-}=\frac{q}{4 \pi \mu_{0} \sqrt{\mu_{x} \mu_{y} \mu_{z}} \sqrt{r^{\prime 2}+d^{\prime 2}-2 r^{\prime} d^{\prime} \cos \theta^{\prime}}}- \\
& -\frac{q}{4 \pi \mu_{0} \sqrt{\mu_{x} \mu_{y} \mu_{z}} \sqrt{r^{\prime 2}+d^{\prime 2}+2 r^{\prime} d^{\prime} \cos \theta^{\prime}}} \approx \frac{2 q d^{\prime} \cos \theta^{\prime}}{4 \pi \mu_{0} \sqrt{\mu_{x} \mu_{y} \mu_{z}} r^{\prime 2}}
\end{aligned}
$$

where $d^{\prime}=d / \sqrt{\mu_{z}}$ and $\cos \theta^{\prime}=z^{\prime} / r^{\prime}$. 

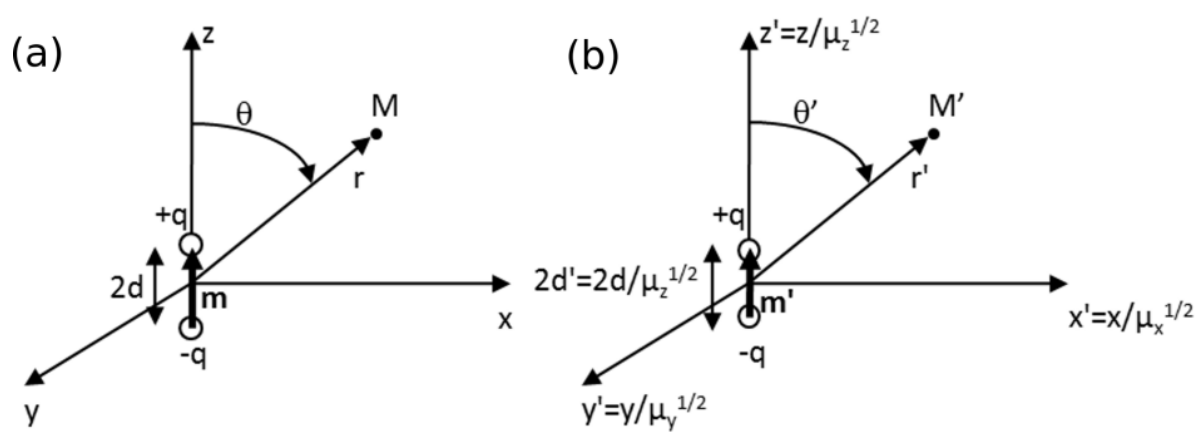

Fig. 5. Definition of the reference frames $(x, y, z),(r, \theta)$ (a) and $\left(x^{\prime}, y^{\prime}, z^{\prime}\right),\left(r^{\prime}, \theta^{\prime}\right)$ (b). This diagram also helps to calculate the magnetostatic potential generated by the dipole moment $\mathbf{m}$ at an arbitrary point $\mathbf{M}$ situated at a distance $r>>d$ from the center of the dipole.

Consider first the simplest case where the ferrofluid chains are in average aligned with the $z$-axis, such that the $x$ and $y$ components of the magnetic permeability are the same: $\mu_{x}=\mu_{y}$. Now, converting Eq. (9) into the original reference frame $(x, y, z)$, introducing polar coordinates $(r, \theta)$, such that $r=\sqrt{x^{2}+y^{2}+z^{2}}$ and $\cos \theta=z / r$, and noting that the absolute value of the dipole moment of a pair of charges is equal to $m=2 q d$, we arrive to the expression:

$$
\phi_{d}=\frac{m}{4 \pi \mu_{0} \mu_{z}^{3 / 2} \mu_{x}} \frac{\cos \theta}{r^{2} g^{3 / 2}}
$$

where $g=\cos ^{2} \theta / \mu_{z}+\sin ^{2} \theta / \mu_{x}$. The components of the intensity of the magnetic field $\mathbf{H}_{1}$ induced by a point dipole $m_{1}$ are found by straightforward calculations:

$$
\begin{aligned}
& H_{1 r}=-\frac{\partial \phi_{d}}{\partial r}=\frac{m_{1}}{4 \pi \mu_{0} \mu_{z}^{3 / 2} \mu_{x}} \frac{2 \cos \theta}{r^{3} g^{3 / 2}} \\
& H_{1 \theta}=-\frac{1}{r} \frac{\partial \phi_{d}}{\partial \theta}=\frac{m_{1}}{4 \pi \mu_{0} \mu_{z}^{3 / 2} \mu_{x}} \frac{f \sin \theta}{r^{3} g^{3 / 2}}
\end{aligned}
$$


where $f=1+3\left(\mu_{x}^{-1}-\mu_{z}^{-1}\right) \cos ^{2} \theta / g$. Replacing the last expressions in Eq. (6) we get the following formula for the potential energy of interaction between two point dipoles with dipole moments $m_{1}$ and $m_{2}$, as well as the following expressions for the components of the magnetic force between these dipoles:

$$
\begin{gathered}
U=-\left(m_{2} \cos \theta H_{1 r}-m_{2} \sin \theta H_{1 \theta}\right)=-\frac{C}{r^{3}} \frac{2 \cos ^{2} \theta-f \sin ^{2} \theta}{3 h^{3 / 2}}, \\
F_{r}=-\frac{\partial U}{\partial r}=-\frac{C}{r^{4}} \frac{2 \cos ^{2} \theta-f \sin ^{2} \theta}{h^{3 / 2}}, \\
F_{\theta}=-\frac{1}{r} \frac{\partial U}{\partial \theta}=-\frac{C \sin 2 \theta}{r^{4} h^{3 / 2}}\left[\frac{2+f}{3}+\frac{\hat{\mu}-1}{2 h}\left(2 \cos ^{2} \theta-f \sin ^{2} \theta\right)-\frac{\hat{\mu}(\hat{\mu}-1)}{h^{2}} \sin ^{2} \theta\right],
\end{gathered}
$$

where $\hat{\mu}=\mu_{z} / \mu_{x}$ is the anisotropy parameter, $h=\cos ^{2} \theta+\hat{\mu} \sin ^{2} \theta$ and $C=3 m_{1} m_{2} /\left(4 \pi \mu_{0} \mu_{x}\right)$.

The trajectories of the microbeads follow from Eq. (5) giving the following expressions for both components of the force balance:

$$
\begin{aligned}
& \frac{d r}{d t}=\left(b_{1}+b_{2}\right) F_{r} \\
& r \frac{d \theta}{d t}=\left(b_{1}+b_{2}\right) F_{\theta}
\end{aligned}
$$

Eliminating time from Eqs. (13a), (13b), we obtain the equation relating two polar coordinates $r$ and $\theta$ of a pair of moving microbeads:

$$
\frac{1}{r} \frac{d r}{d \theta}=\frac{F_{r}}{F_{\theta}}=\psi(\theta)
$$




$$
\psi(\theta)=\frac{2 \cos ^{2} \theta-f \sin ^{2} \theta}{\sin 2 \theta\left(\frac{2+f}{3}+\frac{\hat{\mu}-1}{2 h}\left(2 \cos ^{2} \theta-f \sin ^{2} \theta\right)-\frac{\hat{\mu}(\hat{\mu}-1)}{h^{2}} \sin ^{2} \theta\right)},
$$

whose solution under the initial condition $r(0)=r_{0}$ and $\theta(0)=\theta_{0}$, reads:

$$
r(\theta)=r_{0} \exp \left(\int_{\theta_{0}}^{\theta} \psi(\theta) d \theta\right)
$$

where the integral is estimated numerically.

The case when microbeads trajectories are close to the $x$ axis (such that the nanoparticle chains are oriented mostly along the $x$-axis) implies that the $y$ and $z$ components of the permeability tensor are equal $\mu_{z}=\mu_{y}$ and this breaks the symmetry of the problem with respect to $\mathrm{z}$ axis. Calculations show that $\mu_{x}$ in the denominator of the formula for the constant $C$ should be replaced by $\left(\mu_{x} \mu_{y}\right)^{1 / 2}$, while the anisotropy parameter (which is always defined as $\hat{\mu}=\mu_{z} / \mu_{x}$ ) should be replaced in all the above formulas by $\hat{\mu} \cos ^{2} \phi+\sin ^{2} \phi$, where $\phi$ is the angle that the projection onto the $x y$-plane of the line connecting the centers of the two microbeads makes with the $x$ axis. Since, in experiments the trajectories are confined into the horizontal $x z$-plain, $\phi=0$ and all the formulas (14), (15) for the bead trajectories remain unchanged.

In the limiting case of an isotropic ferrofluid, $\hat{\mu}=f=h=1$, and the equation of the particle trajectory reads:

$$
\frac{r^{2}}{\cos \theta \sin ^{2} \theta}=\frac{r_{0}^{2}}{\cos \theta_{0} \sin ^{2} \theta_{0}}
$$




\section{Comparison between theory and experiments}

The comparison between theory and experiments for the particle trajectories is shown in Fig 6 for both anisotropic ferrofluid (solid lines) and isotropic ferrofluid with the parameter $\hat{\mu}$ set to unity (dashed lines). The experimental trajectories shown by symbols in Figs.6a,b are rather close to the $z$-axis and, according to the arguments given in the beginning of Sec. IV-C, the bead motion is expected to orient the chains mostly along the $z$-axis. We expect that $\mu_{z}>\mu_{x}$ and, using the semi-empirical value $\hat{\mu}=\mu_{z} / \mu_{x}=1.4$ of the anisotropy parameter [Sec. IV-C], we obtain a rather good agreement between the experimental and theoretical trajectories (solid lines) under assumption of magnetic permeability anisotropy. The experimental trajectories shown in Fig. $6 \mathrm{c}$ are closer to the $x$-axis then to the $z$-axis, and we suppose that the bead motion orients the chains closer to the $x$-direction, the components $\mu_{z}, \mu_{x}$ are interchanged with those for the case of Figs. 6a, b, such that the anisotropy parameter is set to $\hat{\mu}=\mu_{z} / \mu_{x}=1 / 1.4 \approx 0.7$. 

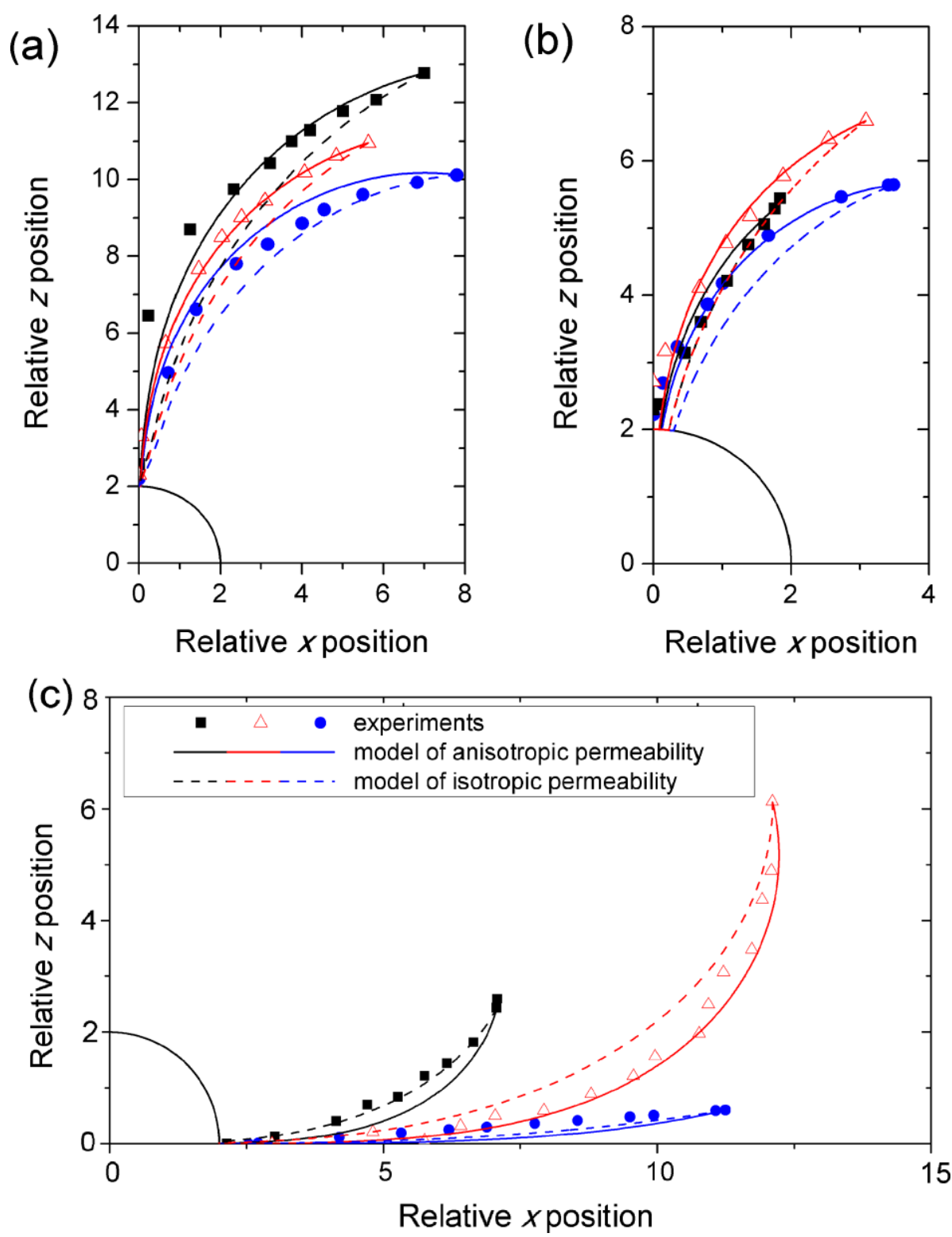

Fig. 6 (Color online). Comparison between the experiments (symbols) and the model for anisotropic (solid lines) or isotropic (dashed lines) ferrofluids predicting the particle trajectories of a pair of $\mathrm{Ni}-\mathrm{Ni}$ (a), PMMA-PMMA (b) and Ni-PMMA microbeads (c). For the anisotropic ferrofluid, the anisotropy parameter $\hat{\mu}$ is set to 1.4 for (a) and (b), and to 0.7 for (c). For the isotropic ferrofluid, $\hat{\mu}=1$ and we use a simple equation (16). The relative particle position $x=r \cdot \sin \theta$ and $z=r \cdot \cos \theta$ is normalized by the mean particle radius $\left(a_{1}+a_{2}\right) / 2$. The external magnetic field is oriented along the $z$-axis. Three different trajectories correspond to three different initial positions of particles.

For the quantitative comparison of performances of both anisotropic and isotropic models, we measured deviations between experiments and both models defined for each experimental point as the shortest distance between a given point and 
the theoretical curve. These deviations are shown and carefully analyzed for each 9 experimental curves in Fig. 9 of Appendix D. Analysis of Fig. 9 shows that the model of anisotropic magnetic permeability gives in general a better prediction of the particle trajectories of pairs of similar microbeads (Ni-Ni and PMMA-PMMA), as compared to the model of isotropic magnetic permeability. The anisotropy model also gives a better prediction for those trajectories of dissimilar microbeads (Ni-PMMA) that are closer to the $x$-axis. A better agreement between experiments and anisotropic model can be explained by the fact that the ratio $F_{z} / F_{x}=d z / d x$ of the magnetic force components $F_{z}$ and $F_{x}$ is a decreasing function of the anisotropy parameter $\hat{\mu}$. This implies that the trajectories close to the $z$-axis with $\hat{\mu}>1$ will shift closer to the $z$-axis with respect to isotropic case, while the trajectories close to the $x$-axis with $\hat{\mu}<1$ will shift closer to the $x$-axis, as observed in experiments.

However, the prediction of the anisotropic model is poorer for the Ni-PMMA pair trajectory that is quite far from both $x$ and $z$ axes (squares in Fig. 6c). Such disagreement could be explained by the fact that the components $\mu_{z}, \mu_{x}$ of the magnetic permeability tensor are expected to be close to each other for the nanoparticle chains oriented along the trajectory denoted by squares, whose direction is more or less in the middle between the directions of both coordinate axes. Thus isotropic permeability should be more appropriate for the calculation of this trajectory. Another particular case arises with that trajectory of the Ni-Ni pair, which is the furthest from the $z$ axis [circles in Fig. 6a]. The initial part of this trajectory is better fitted by the isotropic model and the final part - by the anisotropic model. In fact, at the beginning, the experimental trajectory makes an angle of about $45-60^{\circ}$ with respect to the $z$-axis, which likely results 
in the anisotropy parameter $\hat{\mu}=\mu_{z} / \mu_{x}$ close to unity. At the end of microbeads displacement, the trajectory gets close to the $z$-axis, and the ratio $\hat{\mu}=\mu_{z} / \mu_{x}$ becomes appreciable (1.4 in our model), such that the anisotropic permeability model gives a better prediction.

Finally, it is worth underlying that the single macroscopic physical parameter (apart from initial microbeads position) affecting the microbeads trajectories is the anisotropy parameter, while the magnetic field intensity $H_{0}$, the ferrofluid viscosity, magnetic permeability of the ferrofluid $\left(\mu_{z}, \mu_{x}\right)$ and of microbeads, as well as the microbeads size work out from the ratio $v_{r} / v_{\theta}=F_{r} / F_{\theta}$ of the velocity components appearing in the trajectory equation (14). From the microscopic point of view, the ferrofluid nanoparticle size, or rather the size distribution, is a crucial parameter defining the ferrofluid microstructure and, as a consequence, the anisotropy of the ferrofluid magnetic permeability. As inferred from analysis in Appendix C, larger ferrofluid nanoparticles (or higher volume fraction $\varphi_{L}$ of the largest nanoparticles having a mean diameter $d_{L}$ ) produce larger values of the anisotropy parameter $\hat{\mu}$. Thus the microbeads trajectories will experience a stronger deviation from the isotropic ferrofluid case when increasing nanoparticle size.

\section{E. Kinetics of doublet formation}

To theoretically compute the particle position with time - the function describing kinetics of doublet formation, we can either solve numerically the system of two differential equations (13a), (13b), or obtain a solution in quadratures by substitution of Eq. (15) into Eqs. (13a) and (13b). This last method will give us the following equation: 


$$
t(\theta)=\frac{1}{b_{1}+b_{2}} \int_{\theta_{0}}^{\theta} \frac{r(\theta) d \theta}{F_{\theta}}
$$

where $r(\theta)$ and $F_{\theta}$ are given by Eqs. (15) and (12c), respectively. Equation (17) is applied in its current form if one wants to follow the evolution of the angular position $\theta$ of the approaching microbeads with time. The evolution of the radial position $r$ with time is obtained in a parametric form $(r(\theta), t(\theta))$, where $r(\theta)$ and $t(\theta)$ are given by Eqs. (15) and (17). In order to calculate the time evolution of the microbead positions, we need the expressions for the dipole moments $m_{1}$ and $m_{2}$ of the microbeads in the anisotropic suspending medium (ferrofluid). In the point dipole limit, these expressions read [39]:

$$
m_{i}=\mu_{0}\left(\mu_{i}-\mu_{z}\right) H_{i} v_{i}=\mu_{0}\left(\mu_{i}-\mu_{z}\right) \frac{\mu_{z} H_{0}}{\mu_{z}+n_{z}\left(\mu_{i}-\mu_{z}\right)} v_{i}=4 \pi \mu_{0} \mu_{z} \beta_{i} H_{0} a_{i}^{3}
$$

where subscript " $i$ " refers to either the microbead 1 or the microbead $2, \mu_{i}$ is the microbead magnetic permeability, $v_{i}$ and $a_{i}$ - the microbead volume and radius, $H_{i}$ is the magnetic field intensity inside the microbead, $\beta_{i}=\left(\mu_{i}-\mu_{z}\right) /\left[3\left(\mu_{z}+n_{z}\left(\mu_{i}-\mu_{z}\right)\right)\right]$ is the magnetic contrast factor and $n_{z}$ is the $z$-component of the demagnetizing factor of the oblate ellipsoid of revolution representing the ferrofluid anisotropy and having the semiaxes equal to $\mu_{z}^{-1 / 2}$ and $\mu_{x}^{-1 / 2}$. In the case of the symmetry along the $z$-axis ( $\left.\mu_{z}>\mu_{x}=\mu_{y}, \hat{\mu}>1\right)$ the axis of symmetry of the ellipsoid is oriented along the $z$-axis and $n_{z}=n_{I I}=\left(1+e^{2}\right)(e-\operatorname{atan} e) / e^{3}$ with $e=(\hat{\mu}-1)^{1 / 2}$. In the case of the permeability symmetry along the $x$-axis $\left.\left(\mu_{x}>\mu_{z}=\mu_{y}, \hat{\mu}<1\right)\right)$, the axis of symmetry of the ellipsoid is along the $x$-axis and $n_{z}=\left(1-n_{I I}\right) / 2=1 / 2-\left(1+e^{2}\right)(e-\operatorname{atan} e) /\left(2 e^{3}\right)$ with $e=(1-\hat{\mu})^{1 / 2}$ 
. Thus, the constant $C$ intervening into Eqs. (12b) and (12c) for the magnetic force reads: $C=12 \pi \mu_{0} \mu_{z}^{2} \beta_{1} \beta_{2} a_{1}^{3} a_{2}^{3} H_{0}^{2} /\left(\mu_{x} \mu_{y}\right)^{1 / 2}$ with $\mu_{y}=\mu_{x}$ for $\hat{\mu}>1$ and $\mu_{y}=\mu_{z}$ for $\hat{\mu}<1$.
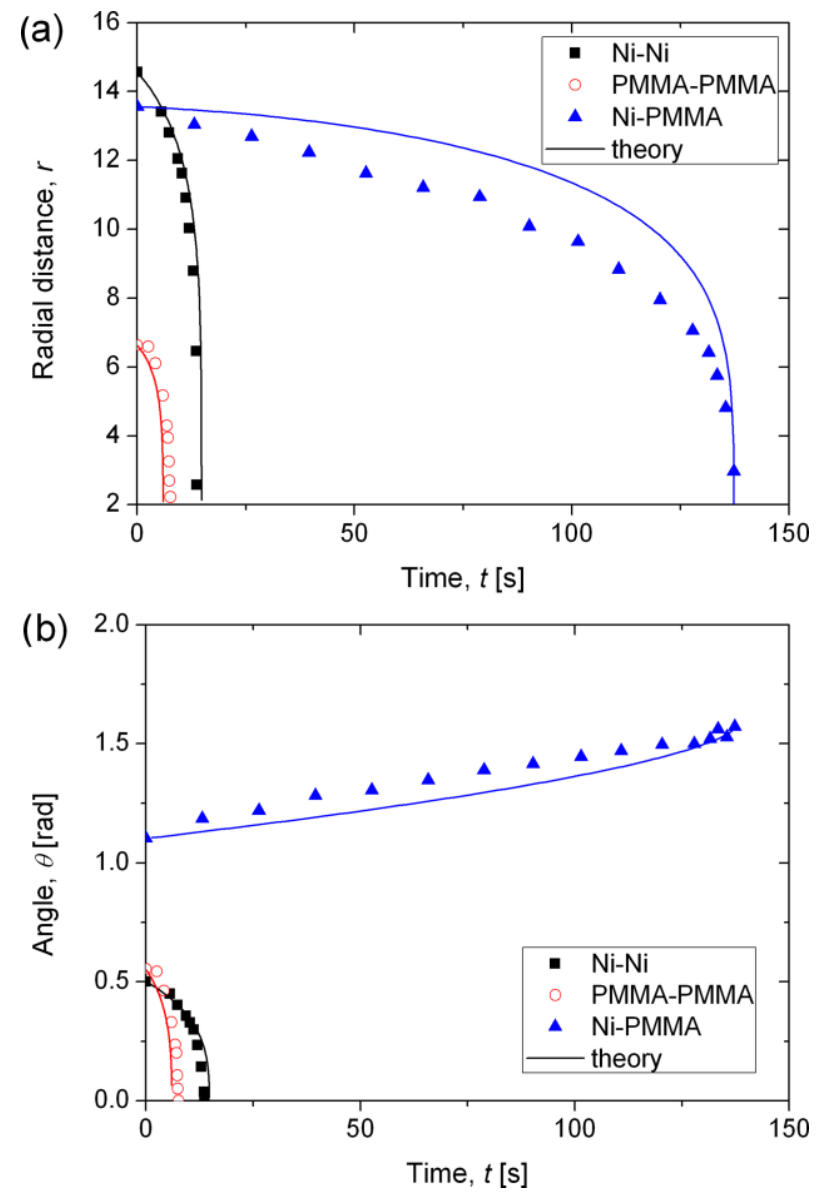

Fig. 7 (Color online). Comparison between experiments (symbols) and theory (lines) for the anisotropic ferrofluid of the microbead radial position (a) and angular position (b) as a function of time. For the pairs of similar microbeads ( $\mathrm{Ni}-\mathrm{Ni}$ and PMMA-PMMA), the anisotropy parameter is equal to $\hat{\mu}=1.4$. For the pair of dissimilar microbeads (Ni-PMMA), the anisotropy parameter is $\hat{\mu}=0.7$. The viscosity is taken to be $0.14 \mathrm{~Pa} \times \mathrm{s}$ for all the curves and is about three times the one of the bulk ferrofluid. The relative radial particle position $r$ in (a) is normalized by the mean microbead radius $\left(a_{1}+a_{2}\right) / 2$.

The comparison between the model and experiments for the particle positions as a function of time is shown in Fig. 7 for the anisotropic ferrofluid with the anisotropy parameter $\hat{\mu}=1.4$ for the trajectories of similar particles and $\hat{\mu}=0.7$ for the trajectories 
of dissimilar particles. Notice that the best fit of the theoretical curves to each set of the experimental data shown on that figure was obtained by setting the viscosity $\eta=0.14 \mathrm{~Pa} \times \mathrm{s}$ of the suspending fluid, which is approximately three times the viscosity of the bulk ferrofluid ( $\eta=0.05 \mathrm{~Pa} \times \mathrm{s})$. This difference can be explained by hydrodynamic interactions between a moving microbead and a bottom wall of the experimental cell, enhancing viscous friction force exerted to the microbead. As observed, the model reproduces reasonably well the time dependencies of the mutual distance and of the mutual angle between microbeads and captures well the fact that Ni-PMMA pair gets much longer time to form a doublet than Ni-Ni or PMAA-PMMA pair (because of longer trajectories). This could be an important conclusion that must be taken into account while considering initial steps of the kinetics of magnetic field-induced particle aggregation.

Let us now briefly inspect the effect of different physical parameters on the time that it will take for two microbeads to approach from their initial positions to the close contact between them, hereinafter called the approach time. According to Eqs. (12c), (17) and the definition of the constant $C$ given below Eq. (18), the approach time $T$ is proportional to $T \propto \eta\left(\mu_{x} \mu_{y} / \mu_{z}{ }^{4}\right)^{1 / 2} /\left(\beta_{1} \beta_{2} a^{5} H_{0}{ }^{2}\right)$ for two microbeads of nearly the same radius $a$. First, one can notice that the approach time increases linearly with the ferrofluid viscosity $\eta$ and decreases with the magnetic field intensity $\left(T \propto H_{0}^{-2}\right)$. Second, the approach time strongly decreases with the bead size, as $T \propto a^{-5}$. This scaling comes from the fact that the time is inversely proportional to the product of the microbeads magnetic moments $m \propto a^{3}$ multiplied by the sum of their hydrodynamic mobilities $\quad b \propto a^{-1}$, namely: $T \propto\left[m_{1} m_{2}\left(b_{1}+b_{2}\right)\right]^{-1}$. Third, for all microbeads 
combinations in the limit of isotropic ferrofluid, the approach time decreases with increasing ferrofluid magnetic permeability $\mu=\mu_{x}=\mu_{y}=\mu_{z}$ under assumption that it remains well below the magnetic permeability $\mu_{i}(i=1$ or 2$)$ of the soft ferromagnetic microbead. This is explained by the fact that the magnetizable medium between microbeads enhances the magnetic force between them [40]. In particular, the response of the soft ferromagnetic microbeads to the applied magnetic field is faster when they are dispersed in a ferrofluid rather than in a nonmagnetic liquid. The same conclusions hold for anisotropic ferrofluid at the considered anisotropy parameters $0.7 \leq \hat{\mu} \leq 1.4$. Finally, at a given transverse component of the magnetic permeability tensor (supposed to be similar to the magnetic permeability $\mu$ of the isotropic ferrofluid [cf. Annex C]) the approach time $T \propto\left(\mu_{x} \mu_{y} / \mu_{z}^{4}\right)^{1 / 2}$ is shown to evolve with the anisotropy parameter as $T \propto \hat{\mu}^{-2}$ for $\hat{\mu}>1$ and $T \propto \hat{\mu}^{-1 / 2}$ for $\hat{\mu}<1$. This implies that the ferrofluid anisotropy accelerates the microbeads motion along the trajectories close to the $z$-axis and decelerates it along the trajectories close to the $x$-axis, with respect to the motion in isotropic ferrofluid.

\section{Conclusions}

We have demonstrated that the anisotropy of the magnetic permeability in ferrofluids strongly affects the magnetostatic force which directs the assembly of microbeads dispersed in the ferrofluid. The magnetic permeability anisotropy stems from the field induced structuring of ferrofluid nanoparticles. Indeed, a theoretical model based on this assumption is able to better describe the experimentally tracked trajectories of pairs of similar (i.e., magnetic-magnetic or non-magnetic-non-magnetic) 
and dissimilar (i.e., magnetic-non-magnetic) particles than a model based on an isotropic approach.

However, if the microbead trajectory strongly deviates from either the direction of the applied magnetic field ( $z$ axis) or transverse direction ( $x$ axis), the model does not capture well the trajectory shape probably because the mutual motion of microbeads generates the ferrofluid chain orientation that induces nearly equal values of the magnetic permeabilities along both coordinate axes, such that the isotropic theory better captures these trajectories. In the case when one trajectory segment strongly deviates from $x$ or $z$ directions and another one comes closer to one of these axes, the isotropic model fits better to the former segment and the anisotropic model - to the latter one. These facts (confirmed by careful comparison of the deviations between experiment and each of both models - see Fig. 9 in Appendix D) suggest that the anisotropy parameter $\hat{\mu}=\mu_{z} / \mu_{x}$ likely varies along the trajectory and is not constant, as assumed in the present model.

We have also found that the multi-polar approach does not significantly improve the theoretical analysis of the particle trajectories. Taken together our results suggest that ferrofluids do not necessarily behave as single phase magnetic continuum in the ferrofluid-directed assembly of microbeads, and that the interactions between the ferrofluid nanoparticles (leading to their structuring) should be taken into account in future theoretical descriptions of this type of assembly.

Apart from particular application to magnetic multi-component suspensions, the present results are expected to contribute to a general understanding of the effects of suspending medium anisotropy on the behavior of suspended particles. 


\section{Acknowledgements}

This work was supported by Project No. FIS2013-41821-R (Ministerio de Economía y Competitividad, Spain; co-funded by ERDF, European Union). P.K. acknowledges financial support of the French government, piloted by the National Research Agency (ANR) in frames of the project Future Investments UCA ${ }^{\mathrm{JEDI}}$, ref $\mathrm{n}^{\circ}$ ANR-15-IDEX-01 (ImmunoMag and RheoGels). A.Z. acknowledges the Russian Fund for Basic Research, (project ref. 16-58-12003), as well as the Program of Russian Federation Ministry of Science and Education (project ref. 3.1438.2017/PCh).

\section{Appendix A. Multipolar theory}

In order to get a more accurate solution than the point-dipole approximation, one needs to consider the multipolar effects of the magnetic interparticle interaction. This requires solution of the standard magnetostatic problem on two magnetizable microbeads placed in the field $H_{0}$ and calculation of magnetic fields inside each of the microbead. To the best of our knowledge, an analytical solution of this problem has not been obtained in literature. A short discussion of the theoretical efforts in this field can be found in [41]. In that work, the energy $U$ of interaction between two identical linearly magnetizable microbeads was calculated by using computer solution of the two-particle problem and the following formula was suggested to fit the numerical results:

$$
U=-3 \mu_{0} \mu_{f} H_{0}{ }^{2} v \sum_{k=3}^{7} \beta^{p_{k}}\left(\frac{a_{k}}{\left(r-b_{k}\right)^{k}}+\frac{c_{k}}{\left(r-d_{k}\right)^{k}} \cos ^{2} \theta\right)
$$

Here $r$ is the distance between microbead centers normalized by their radius, $\theta$ is the angle between the radius-vector $\mathbf{r}$, linking the particle centers, and the applied field $\mathbf{H}_{0}$, $\beta=\left(\mu_{p}-\mu_{f}\right) /\left(\mu_{p}+2 \mu_{f}\right)$ is the magnetic contrast factor where $\mu_{p}$ and $\mu_{f}$ are respectively magnetic permeabilities of the microbead and of the ferrofluid; $v=4 \pi a^{3} / 3$ is 
the microbead volume; $p_{k}, a_{k} \ldots d_{k}$ are some parameters, their values are given in [41]. Note, that for $r>>1$ the formula (A1) coincides with the relation for the point-dipole approximation in the case of the isotropic ferrofluid.

Combining eqs. (1), (2), (5) and (A1), we can calculate the coordinates of the vector $\mathbf{r}$ depending on the time $t$ and determine the particle trajectory in the multipolar approximations. This problem can be solved numerically. The condition $r>2$, which forbids the particle interpenetration, must be taken into account. Results obtained for the multipolar theory did not appreciably differ from the results obtained by considering the point-dipole approximation in the limit of isotropic ferrofluid, as shown in Fig. 8.

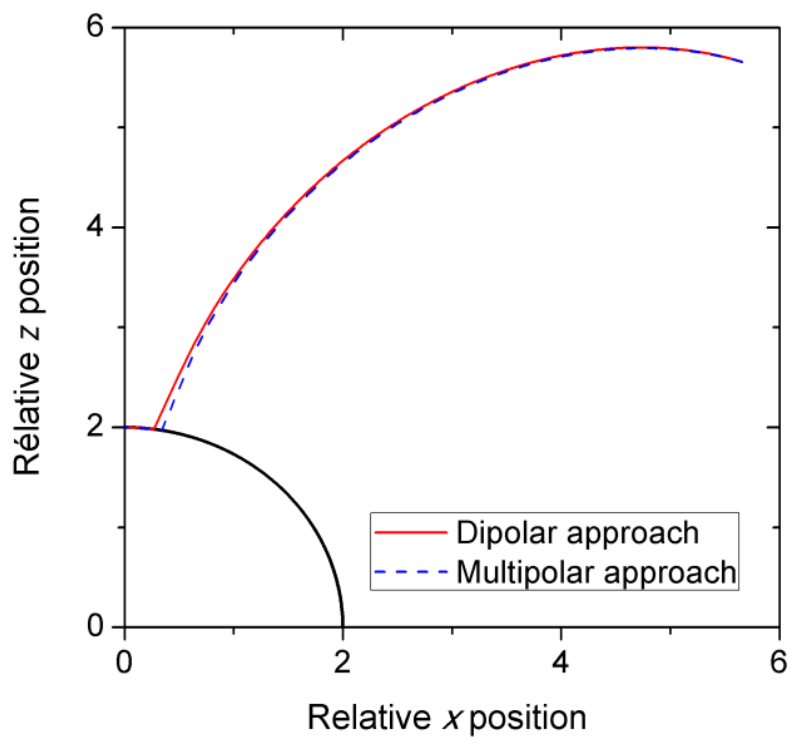

Fig. 8 (Color online). Comparison between multipole (solid line) and point-dipole (dashed line) approaches for calculation of particle trajectories for the Ni-Ni pair of microbeads. The external magnetic field is oriented along the vertical $z$-axis. The relative particle position $x=r \cdot \sin \theta$ and $z=r \cdot \cos \theta$ is normalized by the mean particle radius $\left(a_{1}+a_{2}\right) / 2$.

\section{Appendix B. Equilibrium chain length}

In the case of monodisperse nanoparticles in thermodynamic equilibrium the distribution of the length of nanoparticle chains subject to an external uniform magnetic 
field $H_{0}$ can be calculated by minimizing the free energy of a gas of chains. In the dilute limit and at low field approximation, the following expression for the average number of particles per chain has been obtained using the results of the work [33]:

$$
\begin{aligned}
& \langle N\rangle=\frac{y}{\xi} \frac{1-Y}{Y} \\
& y=\varphi \xi \frac{\exp (2 \lambda)}{12 \pi \lambda^{3}} \\
& Y=\frac{2 y \cosh \xi+\sinh \xi-\sqrt{(2 y \cosh \xi+\sinh \xi)^{2}-4 y^{2}}}{2 y} \\
& \lambda=\frac{m_{n}^{2}}{4 \pi \mu_{0} d^{3} k_{B} T} \\
& \xi=\frac{m_{n} H_{0}}{k_{B} T}
\end{aligned}
$$

where $\varphi$ is the nanoparticle volume fraction, $\lambda$ and $\xi$ are respectively the dipolar coupling and Langevin parameters, with $m_{n}=\pi \mu_{0} M_{S, p} d^{3} / 6$ - the magnetic moment of a ferrofluid nanoparticle and $M_{S, p} \approx 4.05 \times 10^{5} \mathrm{~A} / \mathrm{m}$ - its magnetization taken to be equal to magnetization saturation of magnetite nanoparticles, measured in our previous work [25].

In the case of the model of monodisperse ferrofluid, the nanoparticle diameter, $d$, is supposed to be equal to the average diameter $d_{m}=8.7 \mathrm{~nm}$ of a polydisperse sample. This gives us the average chain length $\langle N\rangle \approx 1.05$, signifying that the nanoparticles of a diameter $d_{m}=8.7 \mathrm{~nm}$ cannot form the chains. This is not surprising because the dipolar 
coupling and Langevin parameters take relatively low values for such a small nanoparticle size: $\lambda\left(d_{m}\right) \approx 0.7$ and $\xi\left(d_{m}\right) \approx 0.4$.

In the case of polydisperse nanoparticle sizes, only the largest particles are supposed to be able to form chains. They have a diameter $d$ larger than some threshold value $d_{0}$ and a high enough value of the dipolar coupling parameter $\lambda$. Since exact analytical theory for magnetic colloids with a continuous size distribution is still not well developed, we propose to model the polydisperse ferrofluid as a bi-disperse system consisting of two nanoparticles fractions: large nanoparticles having equal sizes and forming the chains and small nanoparticles also having equal sizes but remaining isolated. The nanoparticle diameters of both fractions are taken to be equal to the average sizes of polydisperse nanoparticles having diameters $d \geq d_{0}$ and $d \leq d_{0}$ respectively, using the experimental nanoparticle size distribution [Eq. (1), Fig. 1]:

$$
\begin{aligned}
& d_{L}=\int_{d_{0}}^{\infty} x g(x) d x / \int_{d_{0}}^{\infty} g(x) d x \\
& d_{S}=\int_{0}^{d_{0}} x g(x) d x / \int_{0}^{d_{0}} g(x) d x,
\end{aligned}
$$

where the nanoparticle size distribution function $g(d)$ is given by Eq. (1). The volume fraction of large and small nanoparticles is equal to

$$
\begin{gathered}
\varphi_{L}=\varphi \int_{d_{0}}^{\infty} g(x) d x, \\
\varphi_{S}=\varphi \int_{0}^{d_{0}} g(x) d x,
\end{gathered}
$$

respectively, and their sum corresponds to the volume fraction of all ferrofluid nanoparticles: $\varphi_{L}+\varphi_{S}=\varphi$. As inferred from the above formulas, the magnitudes $d_{L}, \varphi_{L}$, 
$d_{S}, \varphi_{S}$ are related to the threshold diameter $d_{0}$ but it is more convenient to relate the magnitudes $\varphi_{L}, d_{S}, \varphi_{S}$ to the diameter of large nanoparticles $d_{L}$, eliminating the auxiliary parameter $d_{0}$. From Eqs. (B-2) and (B-3), we find the following approximate correlation between the volume fraction of large particles and their mean size:

$$
\varphi_{L} \approx \varphi \exp \left(\frac{1-d_{L} / d_{m}}{\sigma}\right),
$$

where $d_{m}=8.7 \mathrm{~nm}$ is the mean diameter of all the ferrofluid particles and $\sigma \approx 0.5$ is the size distribution parameter appearing in Eq. (1). The volume fraction and the diameter of small nanoparticles are are functions of $d_{L}$ and are given by:

$$
\begin{aligned}
& \varphi_{S}=\varphi-\varphi_{L}, \\
& d_{S}=\frac{\varphi d_{m}-\varphi_{L} d_{L}}{\varphi-\varphi_{L}} .
\end{aligned}
$$

In the frame of the above described bi-disperse model, the average chain length of a polydisperse ferrofluid is calculated by previous equations (B-1), in which the volume fraction $\varphi$ of all ferrofluid particles should be replaced by the volume fraction $\varphi_{L}$ of large particles [Eq. (B-4)] and the nanoparticle diameter $d$-by the diameter $d_{L}$ of large particles while calculating the dipolar coupling and Langevin parameters. Fitting the theoretical value of the ferrofluid magnetic permeability [Appendix $\mathrm{C}$ ] to the experimental one, we find the numerical value of $d_{L} \approx 17 \mathrm{~nm}$ and then estimate $\varphi_{L}$ using Eq. (B-4a): $\varphi_{L} \approx 0.15 \varphi \approx 0.01$. Using these numerical values in Eqs. (B-1), we find the average chain length $\langle N\rangle \approx 5.4$ in frames of the bi-disperse model. Note also that the 
dipolar coupling and Langevin parameters take the values $\lambda\left(d_{L}\right) \approx 5.3$ and $\xi\left(d_{L}\right) \approx 3.2$ for the nanoparticles with a diameter $d_{L} \approx 17 \mathrm{~nm}$.

\section{Appendix C. Estimation of the ferrofluid magnetic permeability}

The longitudinal component $\mu_{I I}=\left(\mu_{z}\right.$ or $\left.\mu_{x}\right)$ of magnetic permeability tensor along the nanoparticle chains can be estimated from the equilibrium free energy of the gas of magnetized chains described by a certain length distribution function $f_{N}$. This function has been calculated in [33] along with a volume density $F$ of the free energy in a low-to-moderate field approximation:

$$
\begin{aligned}
& f_{N}=12 \pi \lambda^{3} \exp (-2 \lambda) Y^{N} \\
& F=\frac{6 k_{B} T}{\pi d^{3}} \hat{F} \\
& \hat{F}=\sum_{N=1}^{\infty}\left[f_{N}\left(\ln f_{N}-1\right)+f_{N} Z_{N}\right] \\
& Z_{N}=-\ln \left[\frac{\sinh (N \xi)}{N \xi}(4 \pi)^{N}\left(\frac{\exp (2 \lambda)}{3 \lambda^{3}}\right)^{N-1}\right]
\end{aligned}
$$

where the function $Y$ and the parameters $\lambda$ and $\xi$ are given by Eqs. (B-1c) $-(\mathrm{B}-1 \mathrm{e})$. The longitudinal component of magnetization is proportional to the derivative of the free energy density with respect to the magnetic field intensity; this gives the following expression for the longitudinal component of the magnetic permeability, in the limit of the low magnetic fields:

$$
\mu_{I I}^{c h}=1-48 \lambda\left(\frac{\partial \hat{F}}{\partial \xi^{2}}\right)_{T, f_{N}} \approx 1+96 \pi \lambda^{4} \exp (-2 \lambda) \frac{X(1+X)}{(1-X)^{3}}
$$




$$
X=\frac{1+\varphi \exp (2 \lambda) /\left(6 \pi \lambda^{3}\right)-\sqrt{1+\varphi \exp (2 \lambda) /\left(3 \pi \lambda^{3}\right)}}{\varphi \exp (2 \lambda) /\left(6 \pi \lambda^{3}\right)}
$$

The last formula applies when all the ferrofluid particles form the chains. In the real case of a polydisperse ferrofluid, we consider it as a binary mixture of chains constituted by large nanoparticles with an unknown diameter $d_{L}$ and of isolated nanoparticles with a diameter $d_{S}$ [see Appendix B]. The realistic value of $\mu_{I I}$ for the polydisperse sample is estimated using a mixture rule:

$$
\begin{gathered}
\mu_{I I}=\mu_{I I}^{c h} \frac{\varphi_{L}}{\varphi}+\mu_{i s o}\left(1-\frac{\varphi_{L}}{\varphi}\right) \\
\mu_{i s o} \approx 1+8 \varphi \lambda
\end{gathered}
$$

where $\mu_{i s o}$ is the magnetic permeability of a dilute isotropic mixture of small isolated nanoparticles. The parameter $\lambda$ for isolated nanoparticles [in Eq. (C-4b)] and for nanoparticles constituting the chains [in Eq. (C3)] is calculated by Eq. (B-1d) in which the particle size $d$ should be replaced by the average sizes $d_{S}$ and $d_{L}$ of small and large nanoparticles, respectively.

Relating $\varphi_{L}$ to $d_{L}$ and $d_{S}$ to $d_{L}$ with the help of Eqs. (B-4), (B-5b), we express the longitudinal component of the ferrofluids magnetic permeability [Eq. (C-4a)] as function of a single unknown parameter $d_{L}$. This parameter is found by fitting the theoretical value of $\mu_{I I}$ to the experimental one $\mu_{I I}=1.9$ found from magnetization measurements [Sec. II]. We obtain $d_{L} \approx 17 \mathrm{~nm}$, so a value approximately equal to two mean diameters $\left(d_{m}=8.7 \mathrm{~nm}\right)$ of all ferrofluids particles. This corresponds to the diameter of small nanoparticles $d_{S} \approx 7.3 \mathrm{~nm}$ and to volume fraction of large and small 
nanoparticles equal to $\varphi_{L} \approx 0.15 \varphi \approx 0.010$ and $\varphi_{S} \approx 0.85 \varphi \approx 0.056$, respectively, as inferred from Eqs. (B-4), (B-5). The value $d_{L} \approx 17 \mathrm{~nm}$ also corresponds to the threshold nanoparticle diameter, separating the size distribution [Fig. 1] to classes of small and large nanoparticles, equal to $d_{0} \approx 13 \mathrm{~nm}$.

It is worth mentioning that the longitudinal component $\mu_{I I}$ of the magnetic permeability is related to the average nanoparticle chain length $\langle N\rangle$. To check the stability of our model towards small variations of $\langle N\rangle$, it is estimated that $10 \%$ variation in the chain length around the nominal value $\langle N\rangle=5.4$ gives about 3\% variation in the anisotropy parameter $\hat{\mu}$. It is checked that this produces negligible deviations of the calculated microbeads trajectories from the one calculated at the nominal values $\hat{\mu}=1.4$ or 0.7 .

The component $\mu_{\perp}$ of the magnetic permeability perpendicular to the chains can be estimated from the simple argument that the magnetic field perpendicular to the chain would induce repulsive interactions between particles and the chain would be disintegrated into individual particles, in that case $\mu_{\perp}$ is estimated by Eq. (C-4b) in which the parameter $\lambda$ should be calculated for the average diameter $d_{m}=8.7 \mathrm{~nm}$ of all the ferrofluid particles. The numerical value of $\mu_{\perp}$ is estimated to be equal to 1.38 . Note that such a destruction scenario should not contradict to the existence of chains mainly oriented along the $x$ axis perpendicular to the applied magnetic field when the microbead trajectories are close to this axis. In fact, in the scale of microbeads, the local magnetic field around them is mostly misaligned with the chains oriented along the streamlines such that the chains are not expected to be destroyed by the magnetic field. 
This has been checked for the shear flow in the vicinity of the microbeads where the local magnetic field is almost perpendicular to the streamlines but a small angle between the chains and the flow and high magnetic permeability of nanoparticles are sufficient to keep the integrity of the chains [cf. estimation at the end of Sec. IV-B].

\section{Appendix D. Analysis of deviations between experimental and theoretical} microbeads trajectories.

The measured deviations $\Delta_{i s o}$ and $\Delta_{\text {aniso }}$ between experimental and both theoretical trajectories are shown for each 9 experimental curves in Fig. 9. Recall that these deviations are defined for each point of the experimental trajectory as the shortest distance between a given point and the theoretical curve [Sec. IV-D]. Increasing experimental point number corresponds to the displacement from the initial trajectory point to the final point at the close contact of microbeads. Note that the symbol map in Fig. 9 is the same as the one in Fig. 6.

Two experimental trajectories of Ni-Ni microbeads, situating closer to the $z$-axis are clearly better described by the anisotropic model giving less deviation with experiments along the whole trajectory (two right plots of Fig. 9a). The beginning of the experimental trajectory of $\mathrm{Ni}-\mathrm{Ni}$ pair situating further from the $z$-axis is better described by the isotropic model, while its final part - by anisotropic model (the left plot of Fig. 9a). Such apparent discrepancy could be explained as follows. According to our model, the bead motion orients the ferrofluid nanoparticle chains (in the vicinity of microbeads) along the fluid streamlines, thus, in average, along the trajectory [cf. Sec. IV-B]. When the microbeads move towards each other, the nanoparticle chain orientation in the vicinity of microbeads and, consequently, the magnetic permeability tensor evolve with 
the microbeads mutual position. In the beginning of motion, the considered trajectory [blue circles in Fig. 6a] makes an angle about $\alpha=45-60^{\circ}$ with respect to the $z$-axis of the external magnetic field. Such chain orientation is expected to give the ferrofluid permeability components, $\mu_{z} \sim \mu_{I I} \sin ^{2} \alpha+\mu_{\perp} \cos ^{2} \alpha$ and $\mu_{x} \sim \mu_{I I} \cos ^{2} \alpha+\mu_{\perp} \sin ^{2} \alpha$ close to each other thus, their ratio $\hat{\mu}=\mu_{z} / \mu_{x}$ is expected to be close to unity, as for the isotropic ferrofluid. This possibly explains why the isotropic permeability model better captures the beginning of this trajectory. At the end of microbead displacement, the trajectory gets close to the field $z$-axis, the ferrofluid chains get mostly aligned with the $z$-axis and the ratio $\hat{\mu}=\mu_{z} / \mu_{x}$ becomes appreciable (1.4 in our model), such that the anisotropic permeability model gives a better prediction for the end of the trajectory.

Two of three experimental trajectories of PMMA-PMMA microbeads are much better described by the anisotropic permeability model (left and right plots of Fig. 9b). Another experimental curve seems to be better described by the isotropic model (middle plot of Fig. $9 \mathrm{~b}$ ) but the difference between deviations $\Delta_{i s o}$ and $\Delta_{\text {aniso }}$ falls, for the most of the points, into the error bars $( \pm 0.1)$ of experimental data.

Concerning the Ni-PMMA pair, experimental trajectory furthest from the $x$-axis (full squares in Fig 6c) is better described by the isotropic model (middle plot of Fig. 9c), while the middle experimental trajectory (closer to the $x$-axis, open triangles in Fig. 6c) is better described by the anisotropic model (right plot of Fig. 9c). In the same manner that for the case of Ni-Ni pair, we expect that the trajectory further from the $x$ axis induces the anisotropy ratio $\hat{\mu}=\mu_{z} / \mu_{x}$ closer to unity (because of the ferrofluid particle chain orientation induced by the bead motion), therefore this trajectory is better described by the isotropic model. The trajectory closer to the $x$-axis induces the ratio 
$\hat{\mu}=\mu_{z} / \mu_{x}$ less then unity ( 0.7 in our case) and is better captured by the anisotropic permeability model. Finally, the difference in deviations $\Delta_{i s o}$ and $\Delta_{\text {aniso }}$ for the third curve shown by closed circles in Fig. 6c (the closest curve to the $x$-axis, also see left plot of Fig. 9c), falls within the error bars of the experimental data.
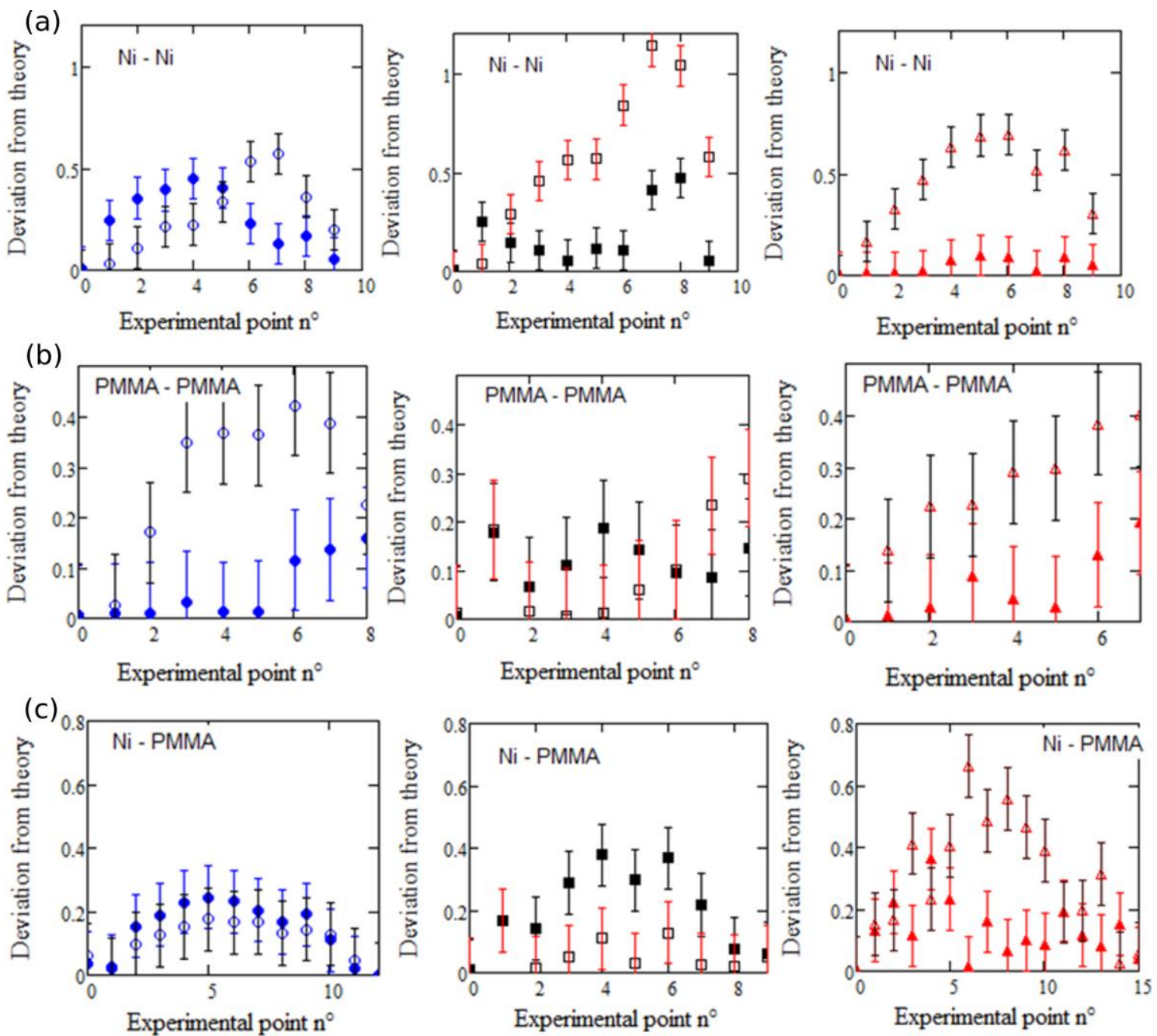

Figure 9 (Color online). Deviation between experimental points of the microbeads trajectories and both theoretical models as function of the number of the experimental point for "Ni-Ni" pair (a), "PMMAPMMA" pair (b) and "Ni-PMMA" pair (c). Closed symbols correspond to the anisotropic model and open symbols - to isotropic model. Three columns correspond to three different trajectories (for each pair of microbeads) shown in Figs. 6a, 6b, 6c. 


\section{References}

1. C. Yu, J. Zhang \& S. Granick. Directed colloidal assembly: Printing with magnets. Nat. Mater. 13, 8-9 (2014).

2. W. Zhao, R. Cheng, J. R. Miller \& L. Mao. Label-Free Microfluidic Manipulation of Particles and Cells in Magnetic Liquids. Adv. Funct. Mater. 26, 39163932 (2016).

3. A.R. Kose, B. Fischer, L. Mao \& H. Koser. Label-free cellular manipulation and sorting via biocompatible ferrofluids. Proc. Natl Acad. Sci. USA, 106, 21478-21483 (2009).

4. R. M. Randall, H. S. Son, B. Samanta, V. M. Rotello \& B. B. Yellen. Magnetic assembly of colloidal superstructures with multipole symmetry. Nature 457, 999-1002 (2009).

5. A. Ray, S. Aliaskarisohi \& T.M. Fischer. Dynamics of self-assembly of flowershaped magnetic colloidal clusters. Phys. Rev. E 82, 031406 (2010).

6. K.H. Li, \& B.B. Yellen. Magnetically tunable self-assembly of colloidal rings. Appl. Phys. Lett. 97, 083105 (2010).

7. K. S. Khalil, A. Sagastegui, Y. Li, M. A. Tahir, J. E. S. Socolar, B. J. Wiley, and B. B. Yellen. Binary colloidal structures assembled through Ising interactions. Nat. Commun. 3, 794 (2012).

8. A. Ray and T. M. Fischer. Magnetic Field Controlled Composite Paramagnetic-Diamagnetic Colloidal Phase. J. Phys. Chem. B, 116, 8233 (2012).

9. A. F. Demirörs, P. P. Pillai, B. Kowalczyk \& B. A. Grzybowski. Colloidal assembly directed by virtual magnetic moulds. Nature 503, 99-10 (2013).

10. Y. Yang, L. Gao, G. P. Lopez \& B. B. Yellen. Tunable Assembly of Colloidal Crystal Alloys Using Magnetic Nanoparticle Fluids. ACS Nano, 7, 2705-2716 (2013).

11. J. Byrom \& S. L. Biswal. Magnetic field directed assembly of two-dimensional fractal colloidal aggregates. Soft Matter, 9, 9167-9173 (2013).

12. M. Wang, L. He \& Y. Yin. Magnetic field guided colloidal assembly. Mater. Today. 16, 110-119 (2013).

13. H. Takahashi, D. Nagao, K. Watanabe, H. Ishii \& M. Konno. Magnetic Field Aligned Assembly of Nonmagnetic Composite Dumbbells in Nanoparticle-Based Aqueous Ferrofluid. Langmuir, 31, 5590-5595 (2015).

14. F. Nogueras-Lara, L. Rodríguez-Arco \& M.T. López-López. Dynamics of magnetic assembly of binary colloidal structures. EPL, 111, 37002 (2015).

15. M.T. López-López, A.Y. Zubarev \& G. Bossis, G. Repulsive force between two attractive dipoles, mediated by nanoparticles inside a ferrofluid. Soft Matter 6, 43464349 (2010).

16. C. Magnet, P. Kuzhir, G. Bossis, A. Meunier, L. Suloeva, \& A. Zubarev. Haloing in bimodal magnetic colloids: The role of field-induced phase separation. Phys. Rev. E 86, 011404 (2012). 
17. C. Magnet, P. Kuzhir, G. Bossis, A. Meunier, S. Nave, A. Zubarev, C. Lomenech \& V. Bashtovoi. Behavior of nanoparticle clouds around a magnetized microsphere under magnetic and flow fields. Phys. Rev. E 89, 032310 (2014).

18. R. W. Ruhwandl \& E. M. Terentjev,. Friction drag on a particle moving in a nematic liquid crystal. Phys. Rev. E 54, 5204 (1996).

19. H. Stark \& D. Ventzki Stokes drag of spherical particles in a nematic environment at low Ericksen numbers. Phys. Rev. E 64, 031711 (2001).

20. V. Hartmann, J. Vermant, E. Heinrich, J. Mewis, \& P. Moldenaers. Effects of particles on the steady state and transient rheology of lyotropic hydroxypropylcellulose solutions. J. Rheol. 44, 1417-1432 (2000).

21. I. I. Smalyukh, A. V. Kachynski, A. N. Kuzmin, \& P. N. Prasad.. Laser trapping in anisotropic fluids and polarization-controlled particle dynamics. Proc. Nat. Acad. Sci. 103, 18048-18053 (2006).

22. F. Mondiot, R. Botet, P. Snabre, O. Mondain-Monval, \& J. C. Loudet. Colloidal aggregation and dynamics in anisotropic fluids. Proc. Nat. Acad. Sci. 111, 5831-5836 (2014).

23. M. Adams, Z. Dogic, S. L. Keller, \& S. Fraden. Entropically driven microphase transitions in mixtures of colloidal rods and spheres. Nature 393, 349 (1998).

24. G. A. Vliegenthart, A. van Blaaderen and H. N. W. Lekkerkerker. Phase transitions, aggregation and crystallization in mixed suspensions of colloidal spheres and rods. Faraday discussions 112, 173-181 (1999).

25. M. T. López-López, J. D. G. Durán, A. V. Delgado \& F. González-Caballero. Stability and magnetic characterization of oleate-covered magnetite ferrofluids in different non-polar carriers. J. Colloid Interface Sci., 291, 144-151 (2005).

26. R. E. Rosensweig, Ferrohydrodynamics (Cambridge University Press, Cambridge, U.K., 1985).

27. D. Jiles, Introduction to Magnetism and Magnetic Materials (Chapman and Hall, London, 1991).

28. M.T. Lopez-Lopez, P. Kuzhir, S. Lacis, G. Bossis, F. Gonzalez-Caballero \& J.D.G. Duran. Magnetorheology for suspensions of solid particles dispersed in ferrofluids. J. Phys. Cond. Matter 18, S2803-S2813 (2006).

29. B. J. de Gans, H. Hoekstra \& J. Mellema. Non-linear magnetorheological behaviour of an inverse ferrofluid Faraday Discuss. 112, 209-224 (1999).

30. L. Rodriguez-Arco, M.T. Lopez-Lopez, A.Y. Zubarev, A. Gdula \& J.D.G. Duran. Inverse magnetorheological fluids, Soft Matter 10, 6256-6265 (2014).

31. S. Odenbach \& H. W. Müller. Stationary off-equilibrium magnetization in ferrofluids under rotational and elongational flow, Phys. Rev. Lett. 89, 037202 (2002).

32. S. Altmeyer, A. Leschhorn, C. Hoffmann, \& M. Lücke. Elongational flow effects on the vortex growth out of Couette flow in ferrofluids. Phys. Rev. E 87, 053010 (2013).

33. A. Yu. Zubarev and L. Yu. Iskakova, Phys. Rev. E 65, 061406 (2002).

34. A. P. Hynninen and M. Dijkstra, Phys. Rev. Lett. 94, 138303 (2005). 
35. R. G. Larson, The Structure and Rheology of Complex Fluids (Oxford University, New York, 1999).

36. J. E. Martin, and R. A. Anderson, "Chain model of electrorheology,' J. Chem. Phys. 104, 4814-4827 (1996)

37. G.K. Batchelor. Brownian diffusion of particles with hydrodynamic interaction. J. Fluid Mechanics 74, 1-29 (1976).

38. G.K. Batchelor \& J.T. Green. The hydrodynamic interaction of two small freelymoving spheres in a linear flow field. J. Fluid Mechanics 56, 375-400 (1972).

39. L.D. Landau \& E.M. Lifshitz. Electrodynamics of continuous media. Pergamon, New York (1984).

40. M. T. López-López, P. Kuzhir, S. Lacis, G. Bossis, F. González-Caballero, \& J.D.G. Durán. Magnetorheology for suspensions of solid particles dispersed in ferrofluids, J. Phys. Condensed Matt. 18, S2803 (2006).

41. A. M. Biller, O. V. Stolbov \& Yu. L. Raikher. Modeling of particle interactions in magnetorheological elastomers. J. Appl. Phys. 116, 114904 (2014). 